\title{
Three-Bladed Rylene Propellers with 3D Network Assembly for Organic Electronics
}

Dong Meng, ${ }^{\dagger, \nabla, \#}$ Huiting Fu, ${ }^{\dagger, \dagger, \nabla, \#}$ Chengyi Xiao, ${ }^{\dagger, \nabla}$ Xiangyi Meng, "Thorsten Winands, ${ }^{\S}$ Wei Ma, ${ }^{\prime}$ Wei Wei, ${ }^{\perp}$ Bingbing Fan, ${ }^{\star}$ Lijun Huo, ${ }^{\star}$ Nikos L. Doltsinis, ${ }^{\S}$ Yan $\mathrm{Li}^{*}{ }^{* \dagger}$ Yanming Sun, ${ }^{*,+}$ and Zhaohui Wang ${ }^{*, \dagger}$

${ }^{\dagger}$ Beijing National Laboratory for Molecular Sciences, Key Laboratory of Organic Solids, Institute of Chemistry, Chinese Academy of Sciences, Beijing 100190, China

${ }^{\dagger}$ Heeger Beijing Research and Development Center, School of Chemistry and Environment, Beihang University, Beijing 100191, China

${ }^{\S}$ Institute for Solid State Theory and Center for Multiscale Theory \& Computation, University of Muenster, Wilhelm-Klemm-Str. 10, 48149 Muenster, Germany

"State Key Laboratory for Mechanical Behavior of Materials, Xi'an Jiaotong University, Xi' an 710049, China

${ }^{\perp}$ Department of Chemistry, Capital Normal University, Beijing 100048, China

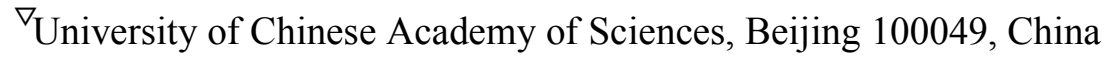

${ }^{\#}$ These authors contributed equally

Table of Contents:

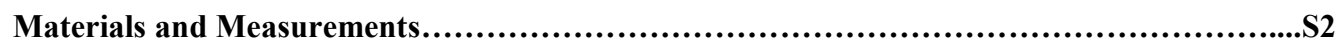

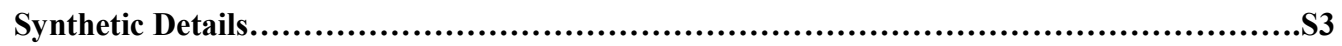

${ }^{1} \mathrm{H}$ NMR Spectra of Compounds 3a, 3b, 4a, 4b, 5a, 6a.............................................................6

${ }^{13}$ C NMR Spectra of Compounds 3a, 3b, 4a, 4b, 5a, 6a........................................S9

TGA curves of compounds $4 \mathrm{a}$ and $6 \mathrm{a}$ and Four stereoisomers of TPH.......................S12

Cyclic voltammetries and DPV profiles of 1a, 4a, 4b, and 6a.............................S13

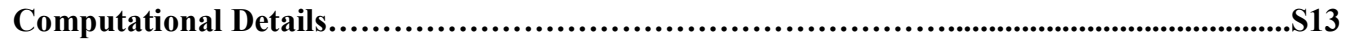

Transistor Device Fabrication and Characterization..............................................................S21

Photovoltaic Device Fabrication and Characterization................................................................S24

References................................................................................... 


\section{Materials and Measurements}

Perylene-3,4,9,10-tetracarboxylicbisimides, 1-bromoperylene-3,4,9,10-tetracarboxyl ic bisimides were synthesized using known procedures. ${ }^{1}$ All chemicals and solvents were purchased from commercial suppliers and used without further purification unless otherwise specified.

${ }^{1} \mathrm{H}$ NMR $(500 \mathrm{MHz})$ and ${ }^{13} \mathrm{C}$ NMR $(125 \mathrm{MHz})$ spectra were recorded in deuterated solvents on a Bruker ADVANCE 500 NMR Spectrometer. $J$ values are expressed in $\mathrm{Hz}$ and quoted chemical shifts are in ppm downfield from tetramethylsilane (TMS) reference using the residual protonated solvent as an internal standard. The signals have been designated as follows: s (singlet), $\mathrm{d}$ (doublet), $\mathrm{t}$ (triplet), q (quartet), sd (singlet doublet), dd (doublet doublet) and $\mathrm{m}$ (multiplets). High resolution mass spectra (HRMS) were determined on IonSpec 4.7 Tesla Fourier Transform Mass Spectrometer.

UV-vis spectra were measured with Hitachi (Model U-3010) UV-vis spectrophotometer in a 1-cm quartz cell. Cyclic voltammograms (CVs) were recorded on a Zahner IM6e electrochemical workstation using glassy carbon discs as the working electrode, $\mathrm{Pt}$ wire as the counter electrode, $\mathrm{Ag} / \mathrm{AgCl}$ electrode as the reference electrode at a scanning rate of $100 \mathrm{mV} / \mathrm{s}$. $0.1 \mathrm{M}$ tetrabutylammoniumhexafluorophosphate $\left(\mathrm{Bu}_{4} \mathrm{NPF}_{6}\right)$ dissolved in $\mathrm{CH}_{2} \mathrm{Cl}_{2}$ was employed as the supporting electrolyte, which was calibrated by the ferrocene/ferroncenium $\left(\mathrm{Fc} / \mathrm{Fc}^{+}\right)$as the redox couple. $\mathrm{CH}_{2} \mathrm{Cl}_{2}$ was freshly distilled prior to use. The microscope images of all the micro-/nano-crystals were acquired by an optical microscope (Vision Engineering Co., UK), which was coupled to a CCD camera. AFM measurements were carried out with a Nanoscope IIIa instrument (Digital Instruments). (GIWAXS) measurements were carried out in a Xenocs-SAXS/WAXS system with X-ray wavelength of $1.5418 \AA$. The samples were irradiated at a fixed angle of 0.2. The films were made on Si wafer substrates under the same preparation conditions for solar cells. Single crystals data collections were performed at $100 \mathrm{~K}$ and $170 \mathrm{~K}$ for $\mathbf{4 b}$ and $\mathbf{6 a}$ on a SuperNova diffractometer and 
MM007HF Saturn724+ diffractometer, using graphite-monochromated $\mathrm{Cu} \mathrm{Ka}$ radiation $(\lambda=1.54184 \AA)$ and Mo $\mathrm{K} \alpha$ radiation $(0.71073 \AA)$, respectively. Using Olex2, these structures were solved with the ShelXS and refined with the ShelXL-2014 refinement package using Least Squares minimization. Refinement was performed on $F^{2}$ anisotropically for all the non-hydrogen atoms by the full-matrix least-squares method. The hydrogen atoms were placed at the calculated positions and were included in the structure calculation without further refinement of the parameters.

\section{$\underline{\text { Synthetic Details }}$}

Compound 3: A Schlenk flask was charged with 1,3,5-benzenetriboronic acid $\begin{array}{llll}\text { tris(pinacol) } & \text { ester } & (1.0 & \text { eq. }),\end{array}$ 1-bromoperylene-3,4,9,10-tetracarboxylicbisimides (2) (3.5 eq.), THF (20ml/2.0g PBI-Br (2)) and $\mathrm{Na}_{2} \mathrm{CO}_{3}$ aqueous solution (2M, $0.5 \mathrm{~V}$ THF). The mixture was degassed with argon for $15 \mathrm{~min} . \mathrm{Pd}\left(\mathrm{PPh}_{3}\right)_{4}$ (0.12 eq.) was added under an argon atmosphere. The mixture was refluxed for $48 \mathrm{~h}$ and then cooled down to room temperature. The cooled mixture was poured into $1 \mathrm{M} \mathrm{HCl}$ aqueous solution $(1000 \mathrm{ml})$ and stirred for $2 \mathrm{~h}$. The precipitate was collected by vacuum filtration, washed with water and $\mathrm{CH}_{3} \mathrm{OH}$, dried, and purified by column chromatography on silica gel, eluted with petroleum ether/ $\mathrm{CH}_{2} \mathrm{Cl}_{2}(2: 1$ to $1: 1 \mathrm{v} / \mathrm{v})$ to afford 3 as dark red solids.

Compound 3a: Yield: 91\%. ${ }^{1} \mathrm{H}$ NMR (500 MHz, $\left.\mathrm{C}_{2} \mathrm{D}_{2} \mathrm{Cl}_{4}, 373.2 \mathrm{~K}\right) \delta=8.89(\mathrm{~s}, 2$ H), 8.72-8.49 (m, $19 \mathrm{H}), 8.02(\mathrm{~s}, 3 \mathrm{H})$, 5.15-5.06 (m, $6 \mathrm{H}), 2.18-2.15(\mathrm{~m}, 12 \mathrm{H})$, 1.91-1.90 (m, $12 \mathrm{H}), 1.34-1.28$ (m, $72 \mathrm{H}), 0.88-0.80$ (m, $36 \mathrm{H}) ;{ }^{13} \mathrm{C}$ NMR (125 MHz, $\left.\mathrm{C}_{2} \mathrm{D}_{2} \mathrm{Cl}_{4}, 373.2 \mathrm{~K}\right): \delta=163.94,139.29,134.96,134.25,132.66,131.38,129.95$, $129.60,128.91,124.33,123.74,123.40,55.14,54.94,32.56,32.48,31.75,31.72$, 29.71, 26.65, 22.47, 22.44, 13.93, 13.84; HRMS (MALDI(N), 100\%): calcd (\%) for $\mathrm{C}_{144} \mathrm{H}_{162} \mathrm{~N}_{6} \mathrm{O}_{12}: 2167.2256$; found, 2167.2252. 
Compound 3b: Yield: 80\%. ${ }^{1} \mathrm{H}$ NMR (500 MHz, $\mathrm{C}_{2} \mathrm{D}_{2} \mathrm{Cl}_{4}, 373.2 \mathrm{~K}$ ) $\delta=8.80-8.69$ (m, $13 \mathrm{H}), 8.56-8.52$ (d, $J=20 \mathrm{~Hz}, 7 \mathrm{H}), 8.23-8.17$ (m, $1 \mathrm{H}), 8.03$ (s, $3 \mathrm{H}), 5.04-4.99$ $\left(\mathrm{t}, J_{1}=10 \mathrm{~Hz}, J_{2}=15 \mathrm{~Hz}, 12 \mathrm{H}\right) ;{ }^{13} \mathrm{C} \mathrm{NMR}\left(125 \mathrm{MHz}, \mathrm{C}_{2} \mathrm{D}_{2} \mathrm{Cl}_{4}, 373.2 \mathrm{~K}\right): \delta=162.69$, $162.55,162.45,146.96,139.38,135.62,134.84,132.18,132.11,130.30,129.61$, $128.91,128.53,127.99,124.39,123.72,123.39,123.29,122.63,122.41,38.76,38.69$; HRMS (MALDI(N), 100\%): calcd (\%) for $\mathrm{C}_{102} \mathrm{H}_{36} \mathrm{~F}_{42} \mathrm{~N}_{6} \mathrm{O}_{12}$ : 2334.1726; found, 2334.1716.

Compound 4: To a standard photocyclization glassware were added $2 \mathrm{~g}$ compound 3 $(10 \times 200 \mathrm{mg}), 500 \mathrm{ml}$ toluene $(10 \times 50 \mathrm{ml})$ and $5 \mathrm{mg} \mathrm{I}_{2}$. The mixture was illuminated by $500 \mathrm{~W}$ mercury lamp for 24 hours. The mixture was extracted with saturated sodium sulfite solution $(2 \times 500 \mathrm{ml})$ and concentrated under reduced pressure. The crude product was purified by column chromatography on silica gel, eluted with petroleum ether/ $\mathrm{CH}_{2} \mathrm{Cl}_{2}(3: 2$ to $1: 1 \mathrm{v} / \mathrm{v})$ to afford $\mathbf{4}$ as red solids.

Compound 4a: Yield: 93\%. ${ }^{1} \mathrm{H}$ NMR $\left(500 \mathrm{MHz}, \mathrm{C}_{2} \mathrm{D}_{2} \mathrm{Cl}_{4}, 373.2 \mathrm{~K}\right) \delta=9.52(\mathrm{~s}, 3 \mathrm{H})$, $9.50(9.505-9.499)(\mathrm{d}, J=3 \mathrm{~Hz}, 9 \mathrm{H}), 9.23-9.22(\mathrm{~d}, J=5 \mathrm{~Hz}, 6 \mathrm{H}), 5.07-5.04(\mathrm{~m}, 6$ $\mathrm{H}), 2.08-2.06(\mathrm{~m}, 12 \mathrm{H}), 1.87-1.83(\mathrm{~m}, 12 \mathrm{H}), 1.25(\mathrm{~s}, 72 \mathrm{H}), 0.79-0.78(\mathrm{~m}, 36 \mathrm{H}) ;{ }^{13} \mathrm{C}$ NMR $\left(125 \mathrm{MHz}, \mathrm{C}_{2} \mathrm{D}_{2} \mathrm{Cl}_{4}, 373.2 \mathrm{~K}\right): \delta=164.24,163.97,134.62,133.55,130.31$, $129.06,127.48,126.45,125.26,124.45,124.15,123.07,123.02,54.86,32.33,32.19$, 31.55, 31.46, 26.48, 22.41, 22.30, 13.85, 13.80; HRMS (MALDI(N), 100\%): calcd (\%) for $\mathrm{C}_{144} \mathrm{H}_{156} \mathrm{~N}_{6} \mathrm{O}_{12}: 2161.1787$; found, 2161.1774 .

Compound 4b: Yield: $83 \%$. ${ }^{1} \mathrm{H}$ NMR $\left(500 \mathrm{MHz}, \mathrm{C}_{2} \mathrm{D}_{2} \mathrm{Cl}_{4}, 373.2 \mathrm{~K}\right) \delta=9.69$ (s, $\left.6 \mathrm{H}\right)$, 9.64-9.62 (d, $J=10 \mathrm{~Hz}, 6 \mathrm{H}), 9.35-9.34(\mathrm{~d}, J=5 \mathrm{~Hz}, 6 \mathrm{H}), 5.01-4.94\left(\mathrm{t}, J_{1}=15 \mathrm{~Hz}, J_{2}\right.$ $=20 \mathrm{~Hz}, 12 \mathrm{H}) ;{ }^{13} \mathrm{C}$ NMR $\left(125 \mathrm{MHz}, \mathrm{C}_{2} \mathrm{D}_{2} \mathrm{Cl}_{4}, 373.2 \mathrm{~K}\right): \delta=163.23,163.00,135.24$, $134.30,131.15,128.62,127.58,126.90,125.83,124.83,124.73,122.11,121.82$, 38.61; HRMS (MALDI(N), 100\%): calcd (\%) for $\mathrm{C}_{102} \mathrm{H}_{30} \mathrm{~F}_{42} \mathrm{~N}_{6} \mathrm{O}_{12}: 2328.1257$; found, 2328.1262 .

Compound 5a: Compound 4a (1 g, $0.462 \mathrm{mmol})$ was dissolved in $100 \mathrm{ml} \mathrm{CH}_{2} \mathrm{Cl}_{2}$. Then a diluted solution of fuming nitric acid $(3 \mathrm{ml})$ in $\mathrm{CH}_{2} \mathrm{Cl}_{2}(5 \mathrm{ml})$ was added drop 
by drop over a period of $15 \mathrm{~min}$. The reaction mixture was stirred at room temperature for additional $12 \mathrm{~h}$. Next, the mixture was poured into methanol and the precipitate was collected by vacuum filtration, washed with water, dried to afford isomeric compound 5a as an dark red solid (1.06 g, $100 \%)$. ${ }^{1} \mathrm{H}$ NMR (500 MHz, $\mathrm{C}_{2} \mathrm{D}_{2} \mathrm{Cl}_{4}$, $373.2 \mathrm{~K}) \delta=9.67-9.66(\mathrm{sd}, J=5 \mathrm{~Hz}, 1.5 \mathrm{H}), 9.59-9.56\left(\mathrm{dd}, J_{1}=5 \mathrm{~Hz}, J_{2}=10 \mathrm{~Hz}, 3\right.$ H), 9.49-9.48 (sd, $J=5 \mathrm{~Hz}, 1.5 \mathrm{H}), 9.25(\mathrm{~s}, 3 \mathrm{H}), 9.16-9.12\left(\mathrm{q}, J_{1}=5 \mathrm{~Hz}, J_{2}=J_{3}=10\right.$ Hz, $6 \mathrm{H}), 5.04$ (m, $6 \mathrm{H}), 2.06$ (s, $12 \mathrm{H}), 1.85$ (s, $12 \mathrm{H}), 1.24$ (s, $72 \mathrm{H}), 0.79$ (s, $36 \mathrm{H})$; ${ }^{13} \mathrm{C}$ NMR $\left(125 \mathrm{MHz}, \mathrm{C}_{2} \mathrm{D}_{2} \mathrm{Cl}_{4}, 373.2 \mathrm{~K}\right): \delta=163.35,163.05,162.40,148.58,135.02$, $132.83,130.34,129.60,129.56,129.54,129.26,129.23,128.00,127.91,127.87$, $126.98,126.85,126.64,126.51,125.74,125.59,125.36,125.28,125.19,124.79$, $124.70,124.35,123.89,122.61,55.44,55.16,32.06,31.94,31.48,31.45,31.33$, 31.30, 26.34, 22.31, 22.20, 13.76, 13.70; HRMS (MALDI(N), 100\%): calcd (\%) for $\mathrm{C}_{144} \mathrm{H}_{153} \mathrm{~N}_{9} \mathrm{O}_{18}: 2296.1339$; found, 2296.1331.

Compound 6a: Selenium $(670 \mathrm{mg}, 8.49 \mathrm{mmol})$ was dissolved in $N$-methylpyrrolidone (NMP, $\left.35 \mathrm{~mL}, 70{ }^{\circ} \mathrm{C}\right)$, then compound 5a $(650 \mathrm{mg}, 0.283$ mmol) was added under argon. The mixture was heated to $190{ }^{\circ} \mathrm{C}$ with vigorous stirring for $12 \mathrm{~h}$ until the starting material could not be detected by TLC. Upon cooling to room temperature, the reaction mixture was poured into $1 \mathrm{~L}$ of $2 \mathrm{M} \mathrm{HCl}$, then the precipitate was collected by vacuum filtration, washed with water, dried, and purified by column chromatography on silica gel (petroleum ether $/ \mathrm{CH}_{2} \mathrm{Cl}_{2}, 1: 1 \mathrm{v} / \mathrm{v}$ ) to give 6a as an dark red solid (589 mg, $87 \%$ ). ${ }^{1} \mathrm{H}$ NMR (500 MHz, $\mathrm{C}_{2} \mathrm{D}_{2} \mathrm{Cl}_{4}, 373.2$ K) $\delta=9.94(\mathrm{~s}, 6 \mathrm{H}), 9.83(\mathrm{~s}, 6 \mathrm{H}), 5.16(\mathrm{~m}, 6 \mathrm{H}), 2.15-2.09(\mathrm{~m}, 12 \mathrm{H}), 1.95(\mathrm{~m}, 12 \mathrm{H})$, 1.30-1.24 (m, $72 \mathrm{H}), 0.79(\mathrm{~s}, 36 \mathrm{H}) ;{ }^{13} \mathrm{C} \mathrm{NMR}\left(125 \mathrm{MHz}, \mathrm{C}_{2} \mathrm{D}_{2} \mathrm{Cl}_{4}, 373.2 \mathrm{~K}\right): \delta=$ $164.77,164.21,142.05,134.67,130.99,128.94,128.40,127.42,124.18,123.82$, $122.38,122.16,55.03,32.24,31.53,31.39,26.53,22.33,13.78,13.74$; HRMS (MALDI(N), 100\%): calcd (\%) for $\mathrm{C}_{144} \mathrm{H}_{150} \mathrm{~N}_{6} \mathrm{O}_{12} \mathrm{Se}_{3}$ : 2392.8879 ; found, 2392.8878 . 


\section{$\underline{{ }^{1} \mathrm{H} \text { NMR Spectra of } 3 \mathrm{a}, 3 \mathrm{~b}, 4 \mathrm{a}, 4 \mathrm{~b}, 5 \mathrm{a}, 6 \mathrm{a}\left(500 \mathrm{MHz}, \mathrm{C}_{2} \underline{\mathrm{D}}_{2} \underline{\mathrm{Cl}}_{4}, 373.2 \mathrm{~K}\right)}$}
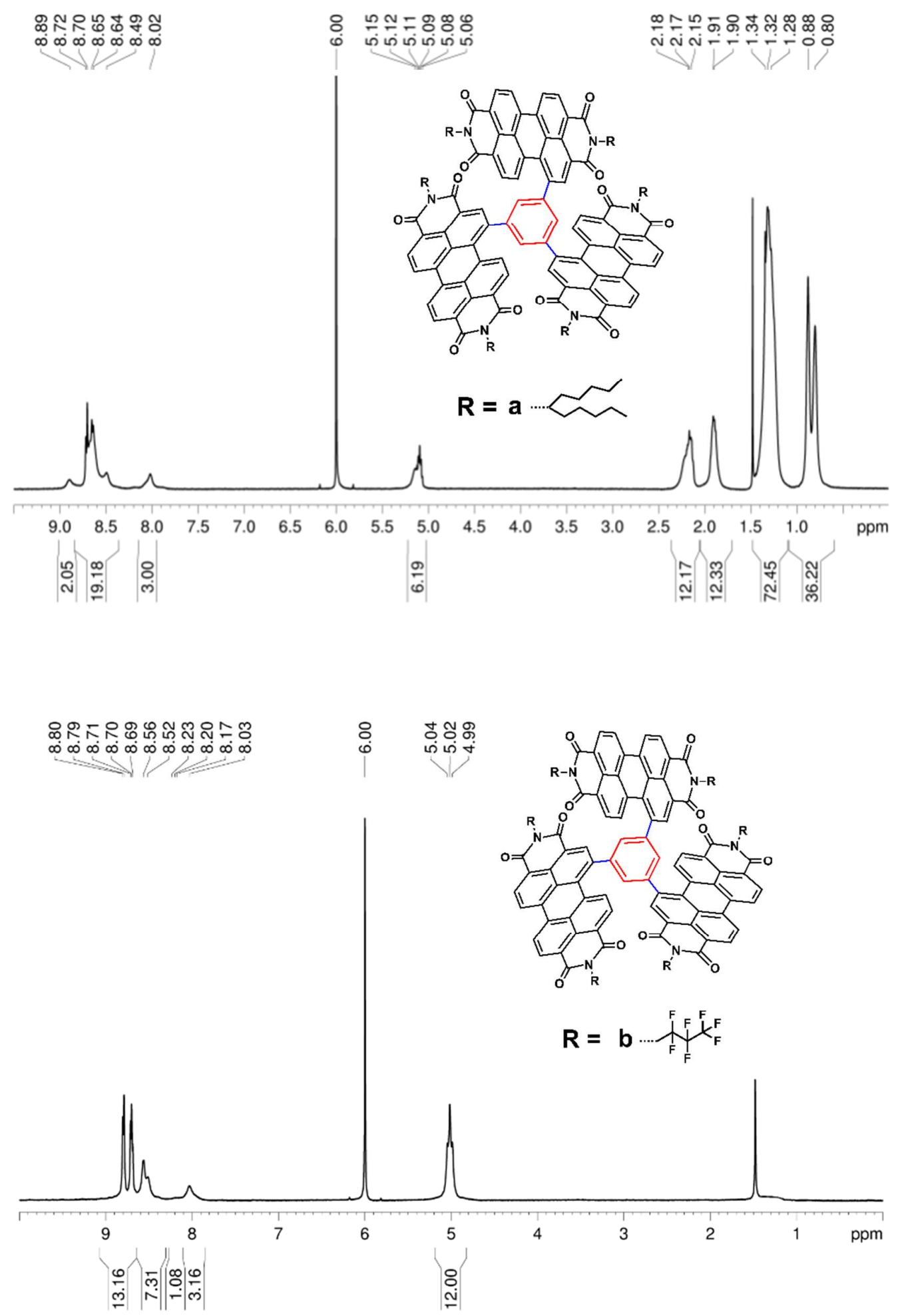

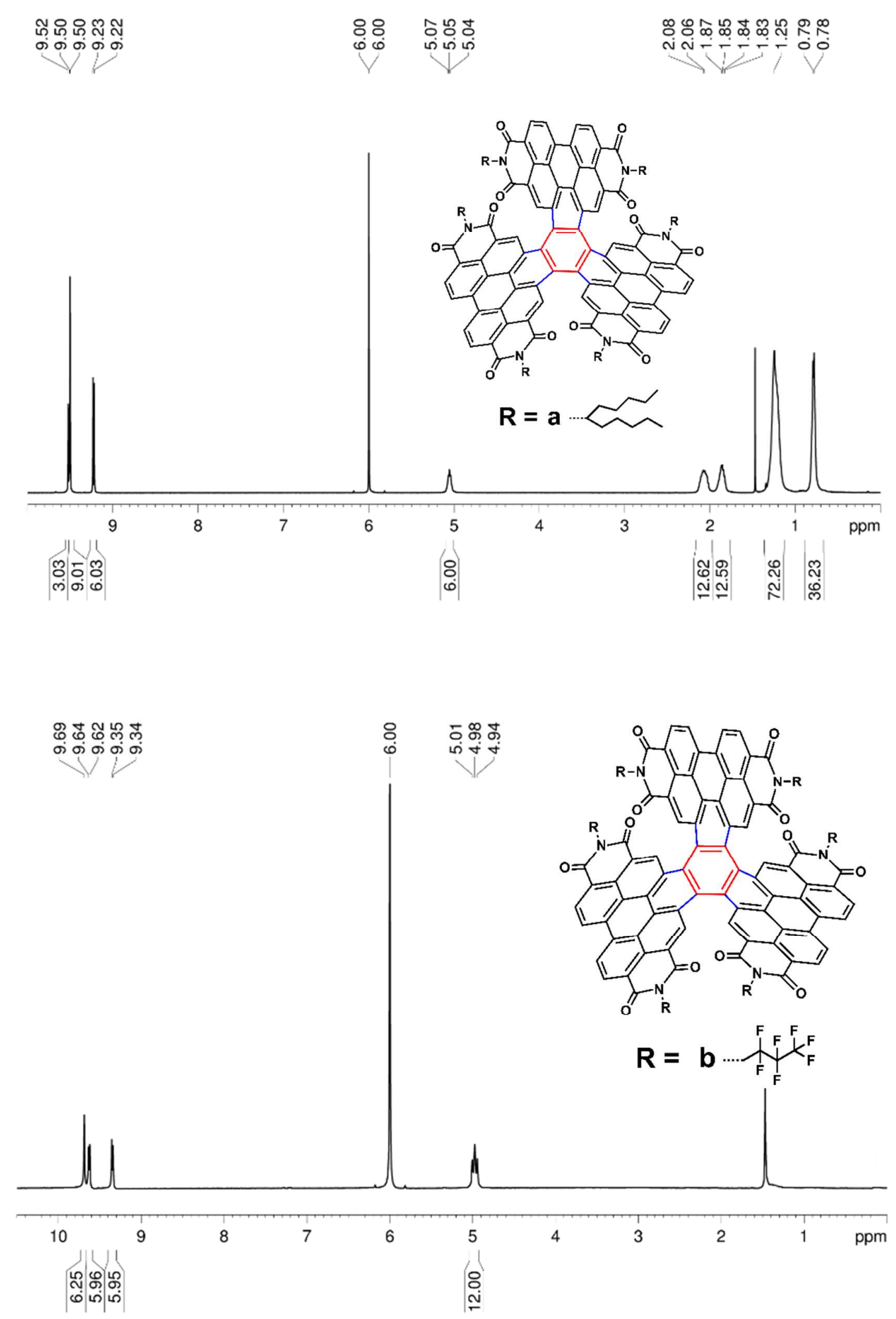

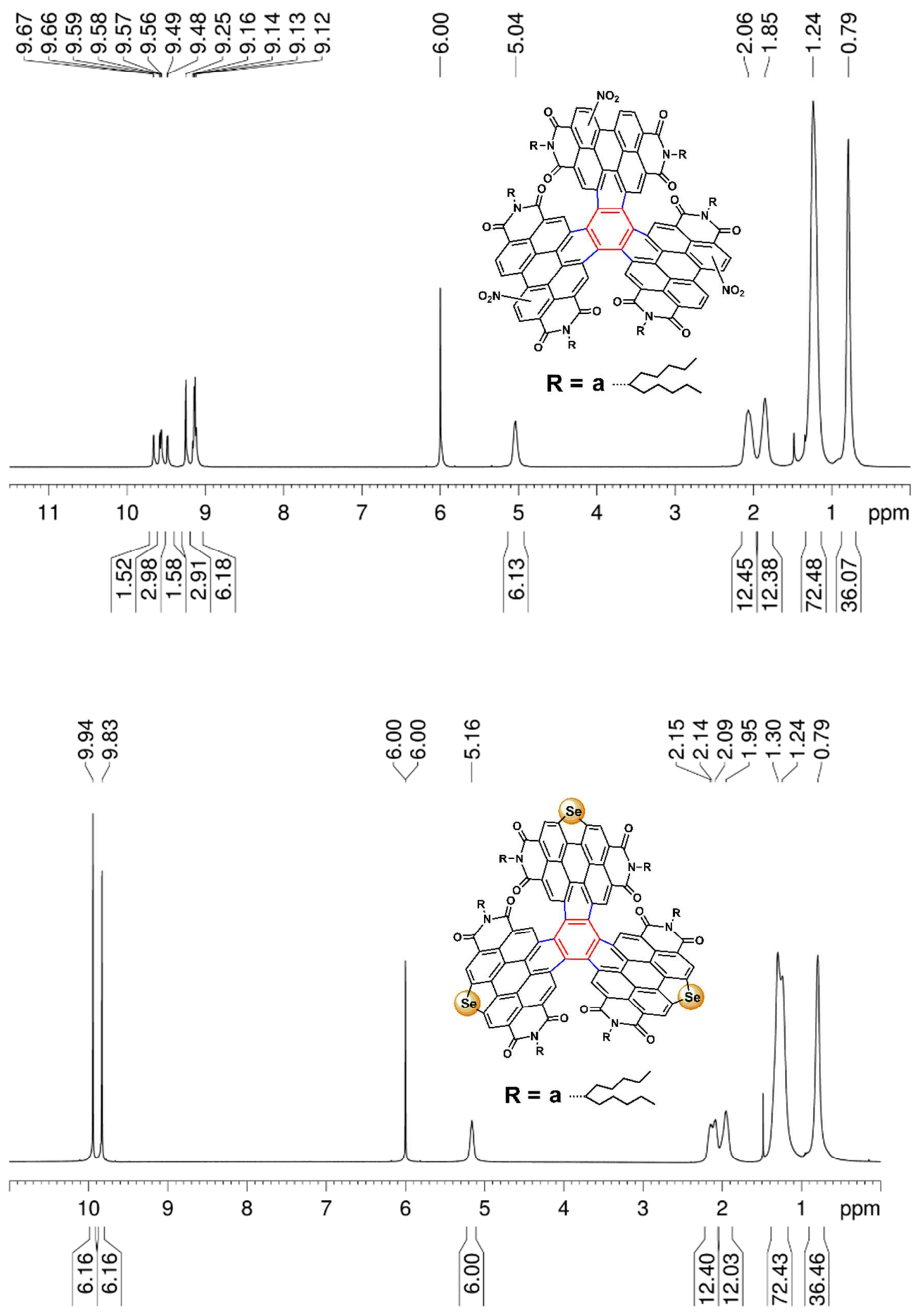
${ }^{13} \mathrm{C}$ NMR Spectra of 3a, 3b, 4a, 4b, 5a, 6a (500 MHz, $\left.\underline{C}_{2} \underline{D}_{2} \underline{\mathrm{Cl}}_{4}, 373.2 \mathrm{~K}\right)$

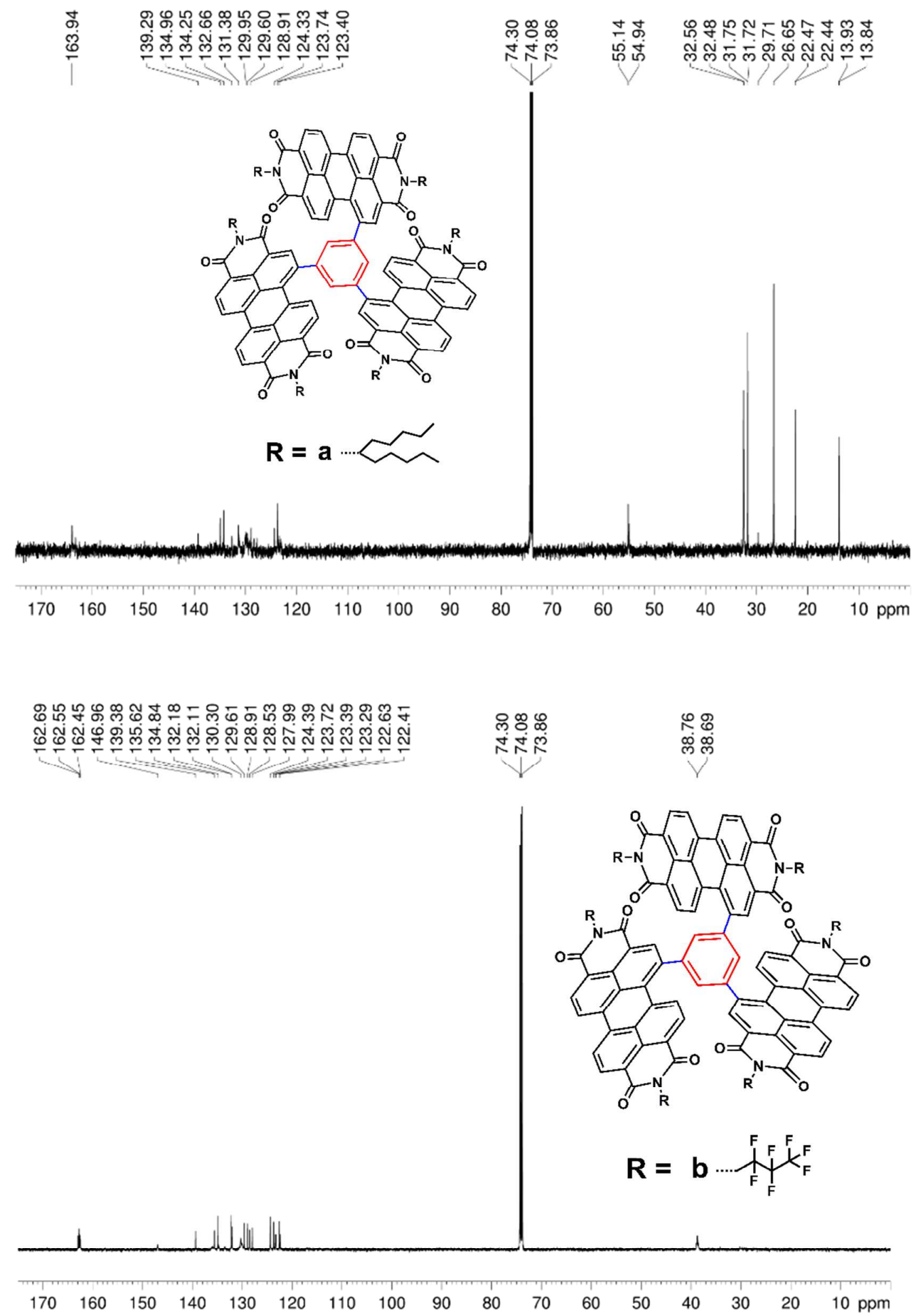



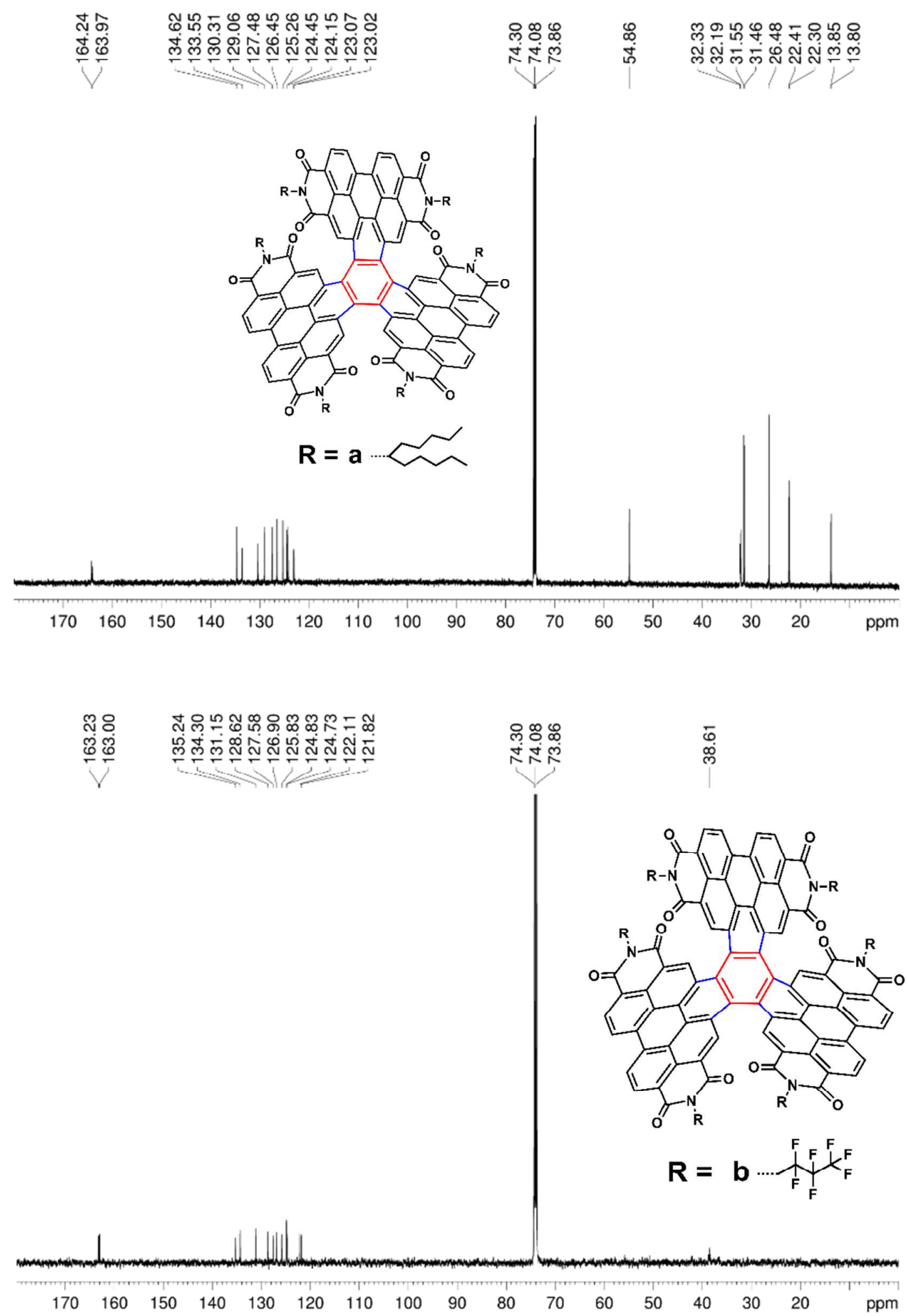


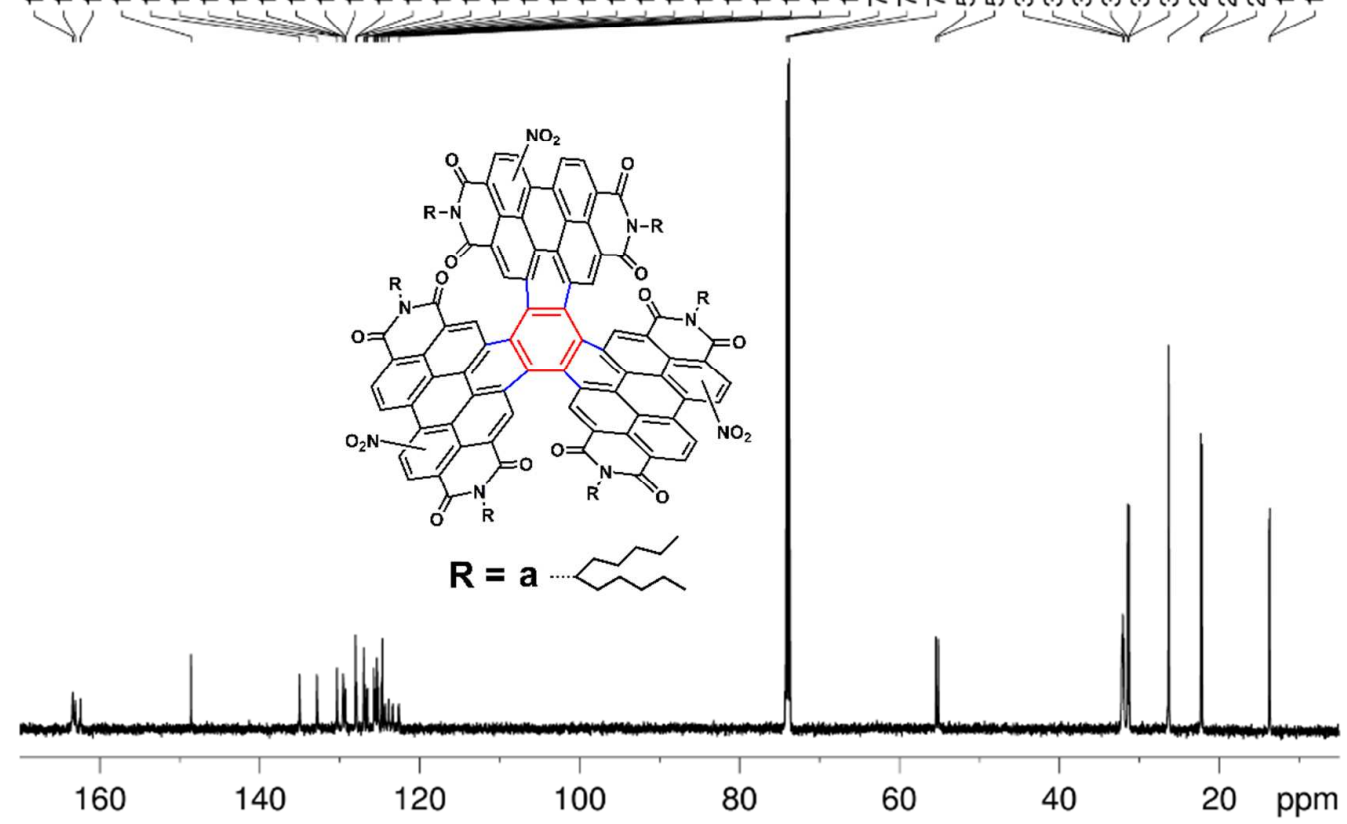

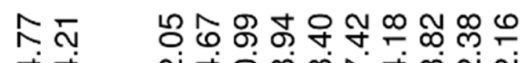

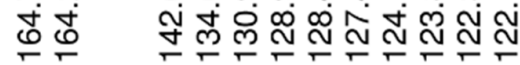
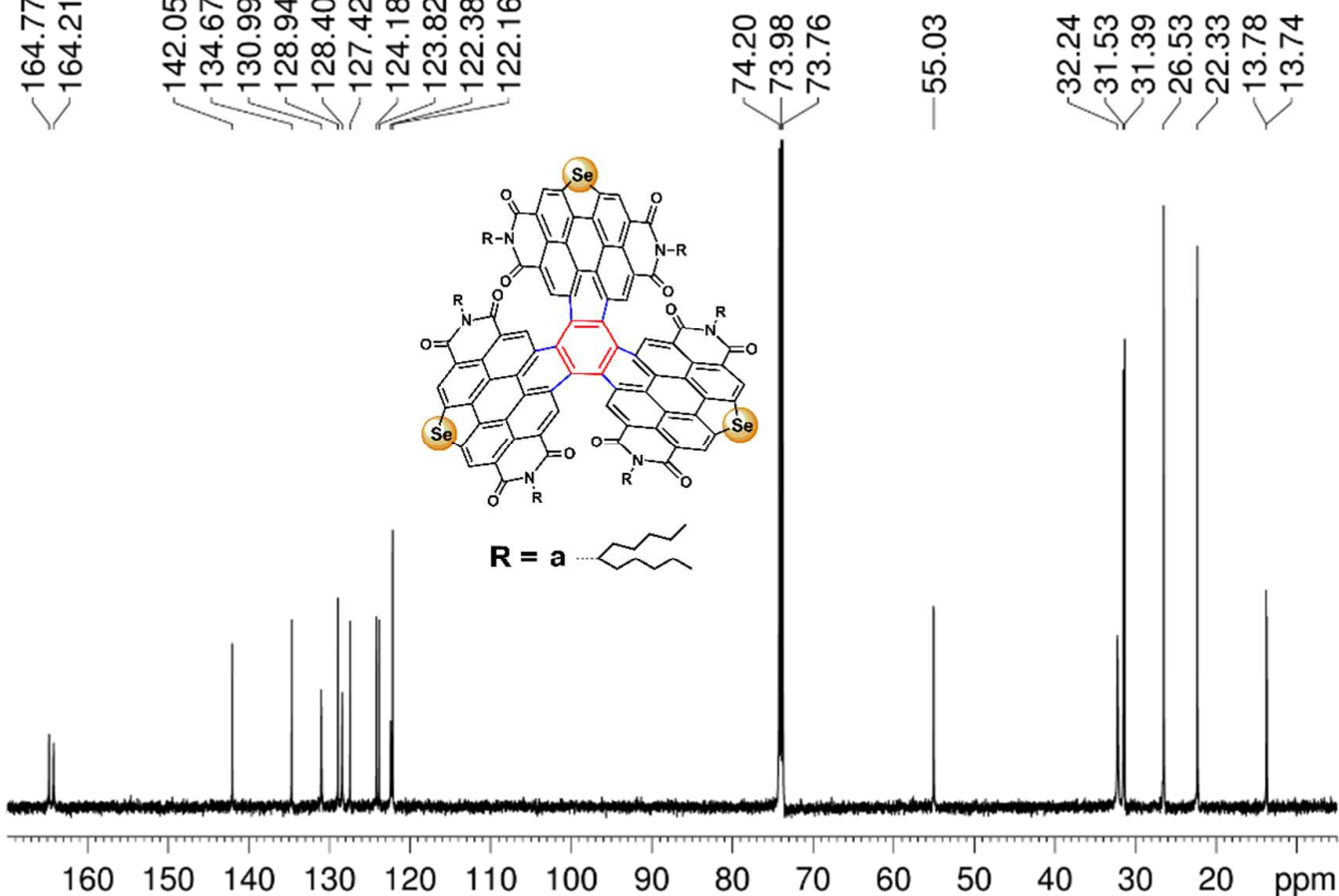
a

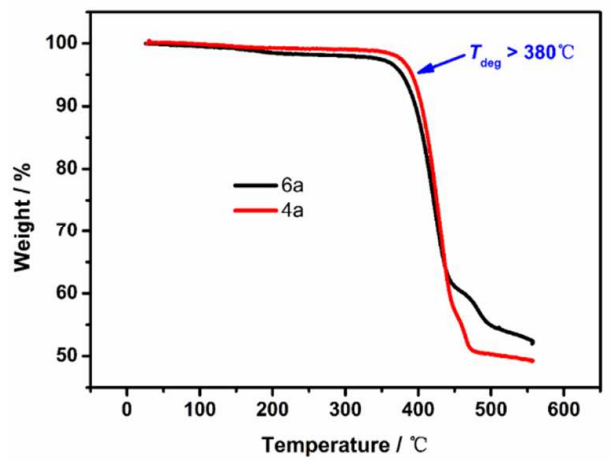

b
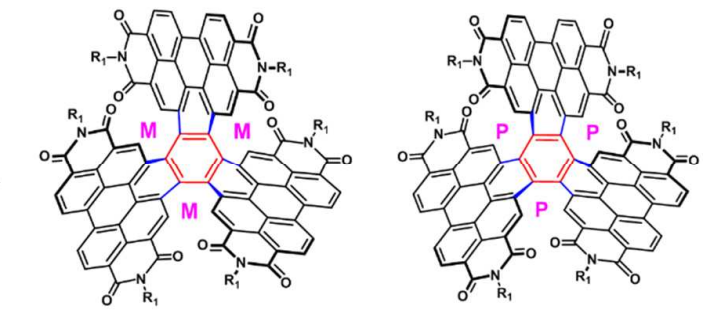

Twist
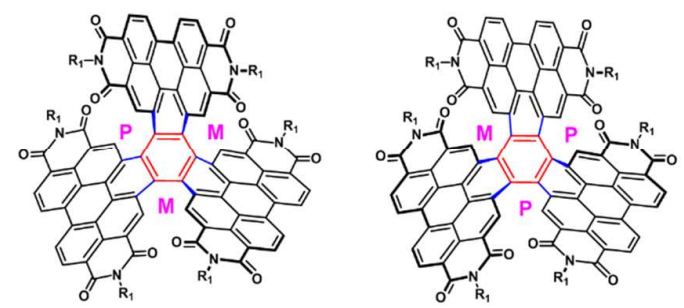

c

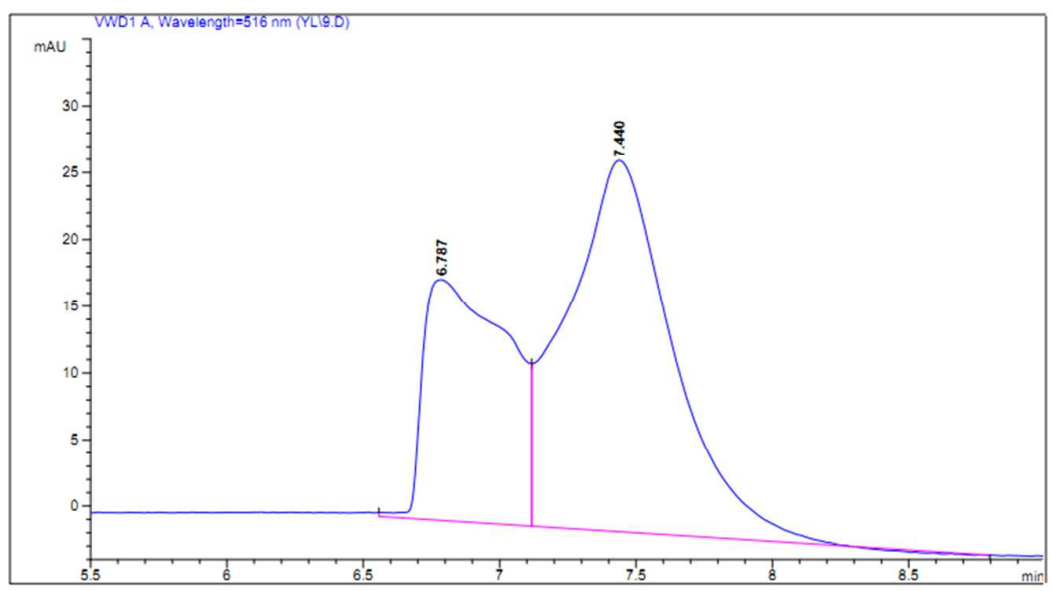

Figure S1. (a) TGA curves of compounds $4 \mathbf{a}$ and $\mathbf{6 a}$ under nitrogen flow, (b) Four stereoisomers of TPH. (c) Chiral HPLC chromatogram of TPH. (Resolution of TPH (Chiralcel OD column; Hex/i-PrOH = 98/2;0.5 mL/min; UV=516 nm)) 

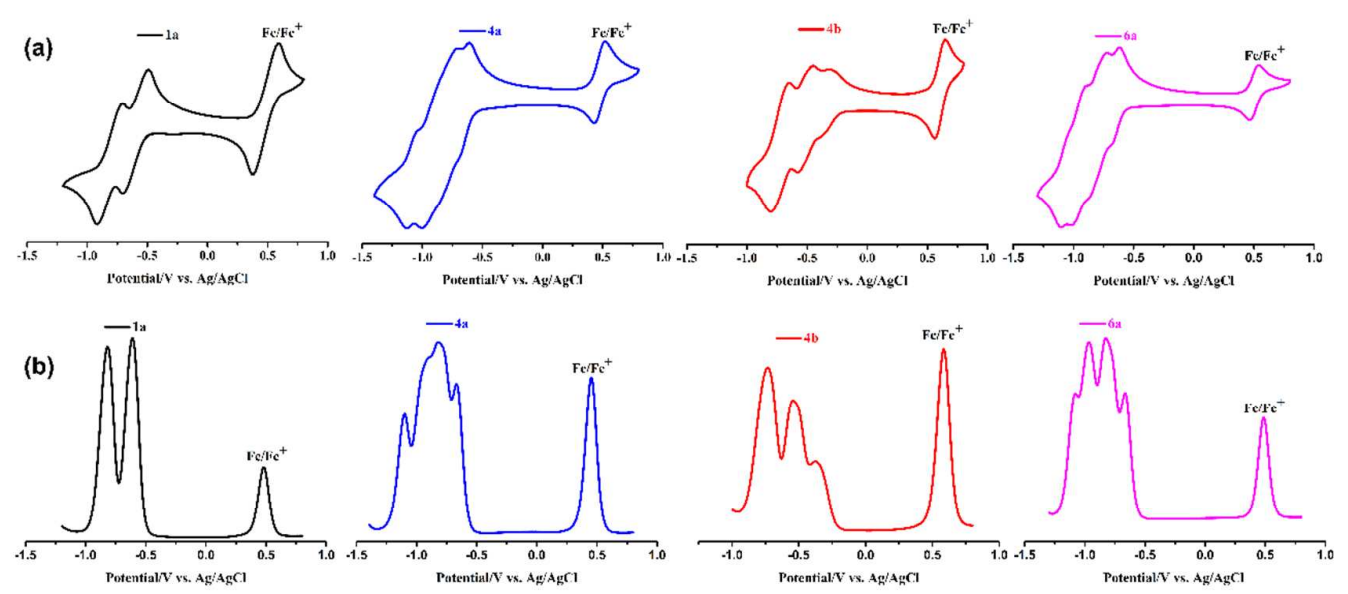

Figure S2. Cyclic voltammetries (a) and DPV profiles (b) of 1a, 4a, 4b and 6a in $\mathrm{CH}_{2} \mathrm{Cl}_{2}$ solution.

\section{Computational Details}

The density functional theory (DFT) calculations were performed with the Gaussian 09 Rev. D.01 quantum chemistry package ${ }^{2}$ employing the PBE0 ${ }^{3}$ exchange-correlation functional and the $6-31 \mathrm{G}^{*}$ basis set. The geometry-optimizations of molecules $1 \mathbf{a}$ and $4 \mathbf{a}$ were performed by using the GEDIIS3 optimization algorithm. ${ }^{4}$ Due to problems reaching the convergence-criteria, the Rational Function Optimization $\left(\mathrm{RFO}^{5}\right)$ algorithm was used for molecules 3a and 6a. In all calculations the chloroform solvent was taken into account using a polarizable continuum model $(\mathrm{PCM})^{6}$ with Bondi atomic radii treating hydrogens explicitly. UV-vis absorption spectras were calculated using time-dependent density functional theory (TDDFT) linear response method. For each molecule, the lowest 80 singlet states were computed. For better comparison with experimental data, Lorentz broadening with a half width at half maximum of $10 \mathrm{~nm}$ was applied to the line spectra obtained. 
Table S1: Eigenvector components of the TDDFT eigenstates for compounds 3a, 4a and $6 \mathbf{a}$ with the highest oscillator strength $\mathrm{f}$.

\section{Compound 3a (HOMO: Orbital 342):}

Excited State 6: Singlet-A $\quad 2.4114 \mathrm{eV} \quad 514.16 \mathrm{~nm} \quad \mathrm{f}=0.2853 \quad<\mathrm{S}^{* * 2}>=0.000$

$$
\begin{array}{ll}
340->343 & -0.30094 \\
340->345 & 0.10515 \\
341->343 & 0.43061 \\
341->344 & 0.44082 \\
342 \text {-> 345 } & 0.10801
\end{array}
$$

Excited State 8: Singlet-A $\quad 2.4537 \mathrm{eV} \quad 505.30 \mathrm{~nm} \quad \mathrm{f}=0.1679 \quad<\mathrm{S} * * 2>=0.000$

$$
\begin{array}{ll}
340->343 & -0.12608 \\
340->344 & 0.52778 \\
340->345 & -0.39590 \\
341->343 & -0.14455 \\
341->344 & 0.12652
\end{array}
$$

Excited State 9: Singlet-A $\quad 2.5189 \mathrm{eV} \quad 492.21 \mathrm{~nm} \quad \mathrm{f}=0.7078 \quad<\mathrm{S} * * 2>=0.000$

$$
\begin{array}{ll}
340->344 & 0.32801 \\
340->345 & 0.52551 \\
341->343 & -0.17360 \\
341->345 & 0.25991
\end{array}
$$

Excited State 10: Singlet-A $\quad 3.2548 \mathrm{eV} \quad 380.92 \mathrm{~nm} \quad \mathrm{f}=0.1306 \quad<\mathrm{S}^{* *} 2>=0.000$

$$
\begin{array}{ll}
338->343 & -0.20748 \\
338->344 & -0.24553 \\
339->343 & 0.53212
\end{array}
$$




$$
339->344 \quad-0.23662
$$

Excited State 12: Singlet-A $\quad 3.2731 \mathrm{eV} \quad 378.80 \mathrm{~nm} \quad \mathrm{f}=0.0421 \quad<\mathrm{S} * * 2>=0.000$

$$
\begin{array}{ll}
338->343 & -0.37477 \\
339->344 & 0.37936 \\
339->345 & 0.37844
\end{array}
$$

Excited State 13: Singlet-A $\quad 3.2849 \mathrm{eV} \quad 377.44 \mathrm{~nm} \quad \mathrm{f}=0.0431 \quad<\mathrm{S}^{* * 2}>=0.000$

$$
\begin{array}{ll}
331->345 & -0.11476 \\
338 \text {-> 343 } & 0.20605 \\
339 \text {-> 344 } & -0.31740 \\
339 \text {-> 345 } & 0.53146
\end{array}
$$

\section{Compound 4a:}

The highest energy peak of compound 4a (HOMO: Orbital 339)

Excited State 18: $\quad$ Singlet-A $\quad 3.2308 \mathrm{eV} \quad 383.75 \mathrm{~nm} \quad \mathrm{f}=0.5668 \quad<\mathrm{S} * * 2>=0.000$

$$
\begin{array}{ll}
338->343 & -0.28176 \\
338->344 & 0.41036 \\
339->343 & 0.36569 \\
339->344 & 0.26872
\end{array}
$$

Excited State 19: $\quad$ Singlet-A $\quad 3.2317 \mathrm{eV} \quad 383.65 \mathrm{~nm} \quad \mathrm{f}=0.5746 \quad<\mathrm{S} * * 2>=0.000$

$$
\begin{array}{ll}
338->343 & -0.34838 \\
338->344 & -0.29369 \\
339->343 & -0.25060 \\
339->344 & 0.42600
\end{array}
$$

Excited State 20: Singlet-A $3.3716 \mathrm{eV} \quad 367.73 \mathrm{~nm} \quad \mathrm{f}=0.3566 \quad<\mathrm{S} * * 2>=0.000$

$$
\begin{array}{ll}
334->340 & 0.21050 \\
335->342 & -0.17131
\end{array}
$$




$$
\begin{array}{ll}
336->342 & -0.12594 \\
337->343 & 0.61201
\end{array}
$$

Excited State 21: $\quad$ Singlet-A $\quad 3.3742 \mathrm{eV} \quad 367.45 \mathrm{~nm} \quad \mathrm{f}=0.3514 \quad<\mathrm{S} * * 2>=0.000$

$$
\begin{array}{ll}
334->341 & 0.21629 \\
335->342 & -0.12580 \\
336->342 & 0.17147 \\
337->344 & 0.60890
\end{array}
$$

Excited State 22: $\quad$ Singlet-A $\quad 3.4890 \mathrm{eV} \quad 355.36 \mathrm{~nm} \quad \mathrm{f}=0.2133 \quad<\mathrm{S}^{* *} 2>=0.000$

$$
\begin{array}{ll}
334->340 & 0.60849 \\
334->341 & -0.14879 \\
337->343 & -0.25498
\end{array}
$$

Excited State 23: Singlet-A $\quad 3.4899 \mathrm{eV} \quad 355.27 \mathrm{~nm} \quad \mathrm{f}=0.2183 \quad<\mathrm{S}^{* *} 2>=0.000$

$$
\begin{array}{ll}
334->340 & 0.14682 \\
334->341 & 0.60614 \\
337 \text {-> 344 } & -0.26092
\end{array}
$$

The lowest energy peak of compound 4a

Excited State 3: $\quad$ Singlet-A $\quad 2.3975 \mathrm{eV} \quad 517.14 \mathrm{~nm} \quad \mathrm{f}=0.7068 \quad<$ S**2 $>=0.000$

$$
\begin{array}{ll}
338->340 & 0.11543 \\
338->341 & 0.46433 \\
339->340 & 0.45703 \\
339->341 & -0.11656 \\
339->342 & 0.16737
\end{array}
$$

Excited State 4: $\quad$ Singlet-A $\quad 2.3980 \mathrm{eV} \quad 517.02 \mathrm{~nm} \quad \mathrm{f}=0.7142 \quad<\mathrm{S}^{* *} 2>=0.000$

$$
\begin{array}{ll}
338->340 & 0.46519 \\
338->341 & -0.11740 \\
338->342 & -0.16563 \\
339->340 & -0.11451 \\
339->341 & -0.45688
\end{array}
$$

\section{Compound 6a:}

The highest energy peak of compound 6a (HOMO: Orbital 387) 
Excited State 21: $\quad$ Singlet-A $\quad 3.1609 \mathrm{eV} \quad 392.24 \mathrm{~nm} \quad \mathrm{f}=0.5485 \quad<\mathrm{S} * * 2>=0.000$

$$
\begin{array}{ll}
386 \text { - } 391 & 0.25693 \\
386 \text { - } 392 & 0.43050 \\
387 \text {-> } 391 & -0.35945 \\
387 \text { - } 392 & 0.27194
\end{array}
$$

Excited State 22: Singlet-A $\quad 3.1613 \mathrm{eV} \quad 392.20 \mathrm{~nm} \quad \mathrm{f}=0.5544 \quad<\mathrm{S} * * 2>=0.000$

$$
\begin{array}{ll}
386 \text { - } 391 & 0.40051 \\
386 \text {-> } 392 & -0.28577 \\
387 \text {-> 391 } & 0.24098 \\
387 \text {-> 392 } & 0.39267
\end{array}
$$

The lowest energy peak of compound 6 a

Excited State 3: $\quad$ Singlet-A $\quad 2.3912 \mathrm{eV} \quad 518.50 \mathrm{~nm} \quad \mathrm{f}=0.8334 \quad<\mathrm{S}^{* *} 2>=0.000$

$$
\begin{array}{ll}
386 \text { - }>388 & 0.44988 \\
386 \text {-> } 390 & 0.12484 \\
387 \text {-> 389 } & 0.44785 \\
387 \text {-> 390 } & -0.22314
\end{array}
$$

Excited State 4: Singlet-A $\quad 2.3913 \mathrm{eV} \quad 518.48 \mathrm{~nm} \quad \mathrm{f}=0.8369 \quad<\mathrm{S} * * 2>=0.000$
$386->389$
0.45647
$386->390$
0.21951
$387->388$
$-0.44228$
$387->390$
0.12674 


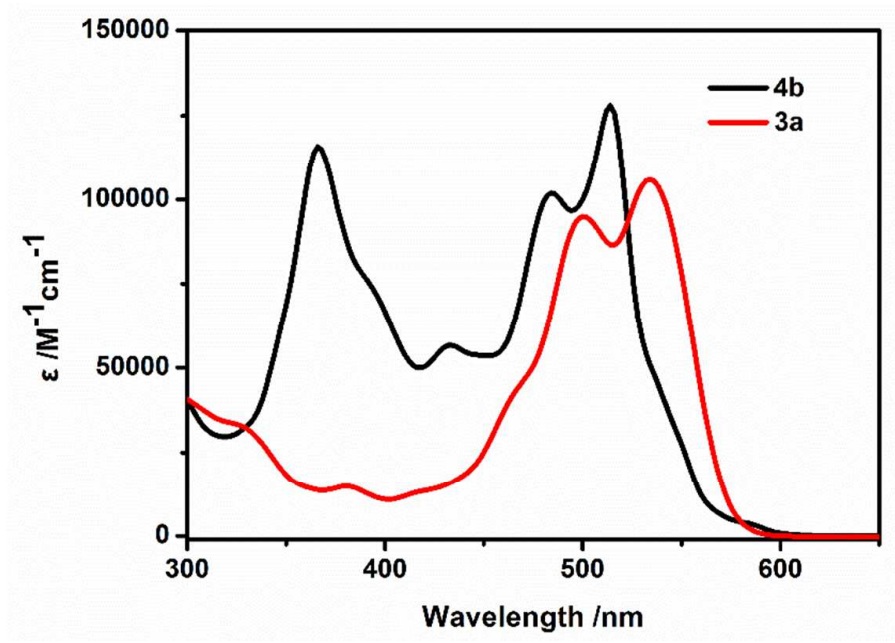

Figure S3. UV-vis absorption spectra of $\mathbf{4 b}$ and $\mathbf{3 a}$ in $\mathrm{CHCl}_{3}\left(1 \times 10^{-5} \mathrm{M}\right)$.

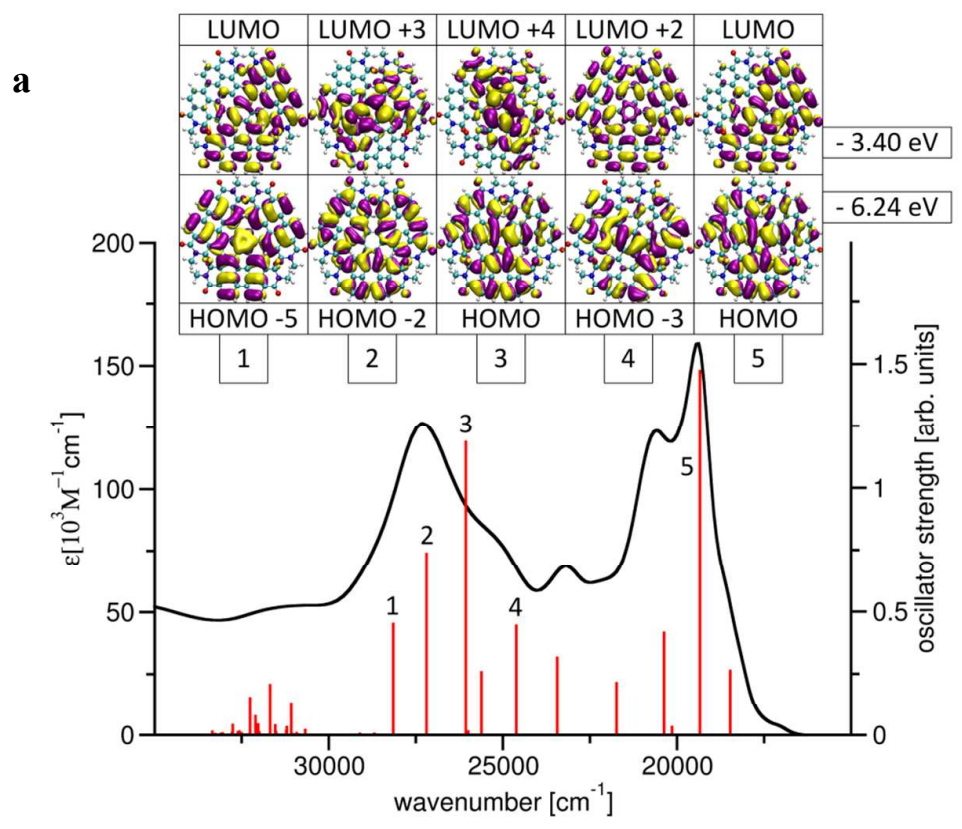




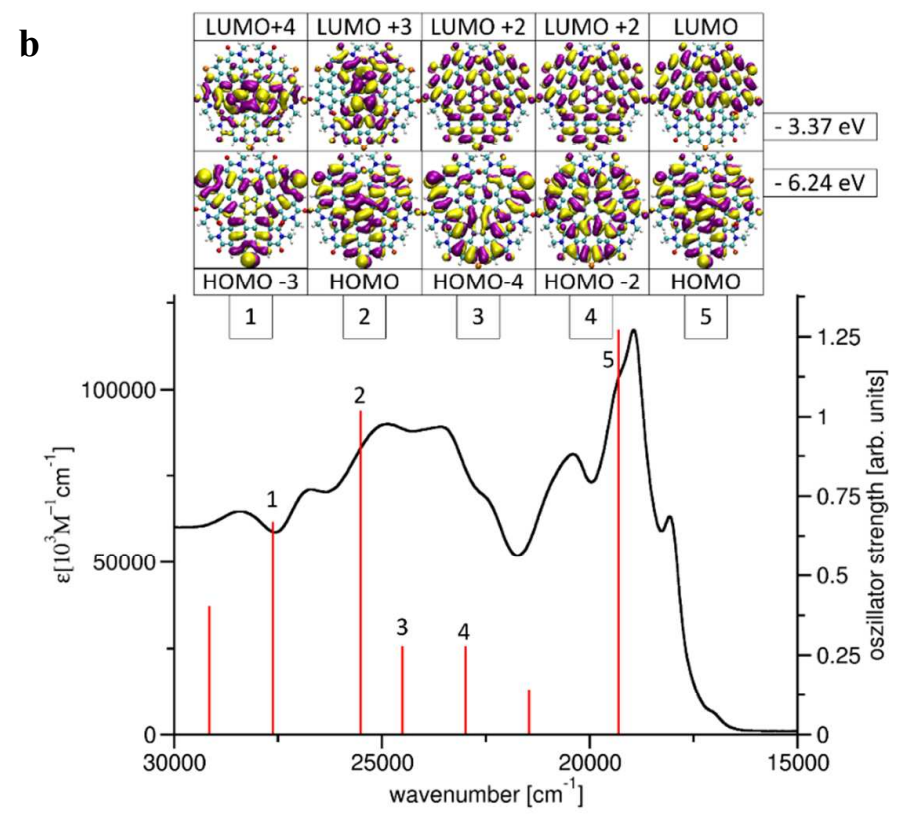

Figure S4. Experimental absorption spectrums (black line) and calculated line spectrum (vertical red lines) of compounds $4 \mathbf{a}(\mathrm{a})$ and $\mathbf{6 a}(\mathrm{b})$.

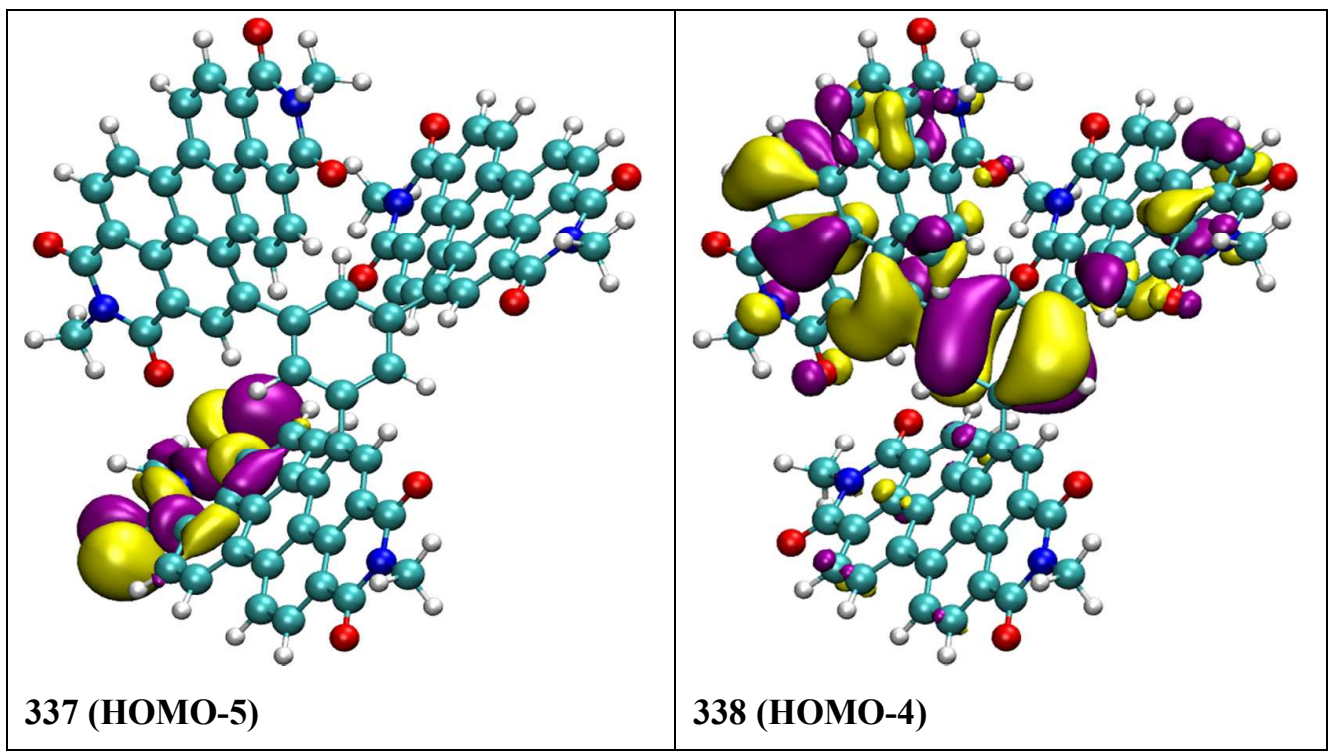




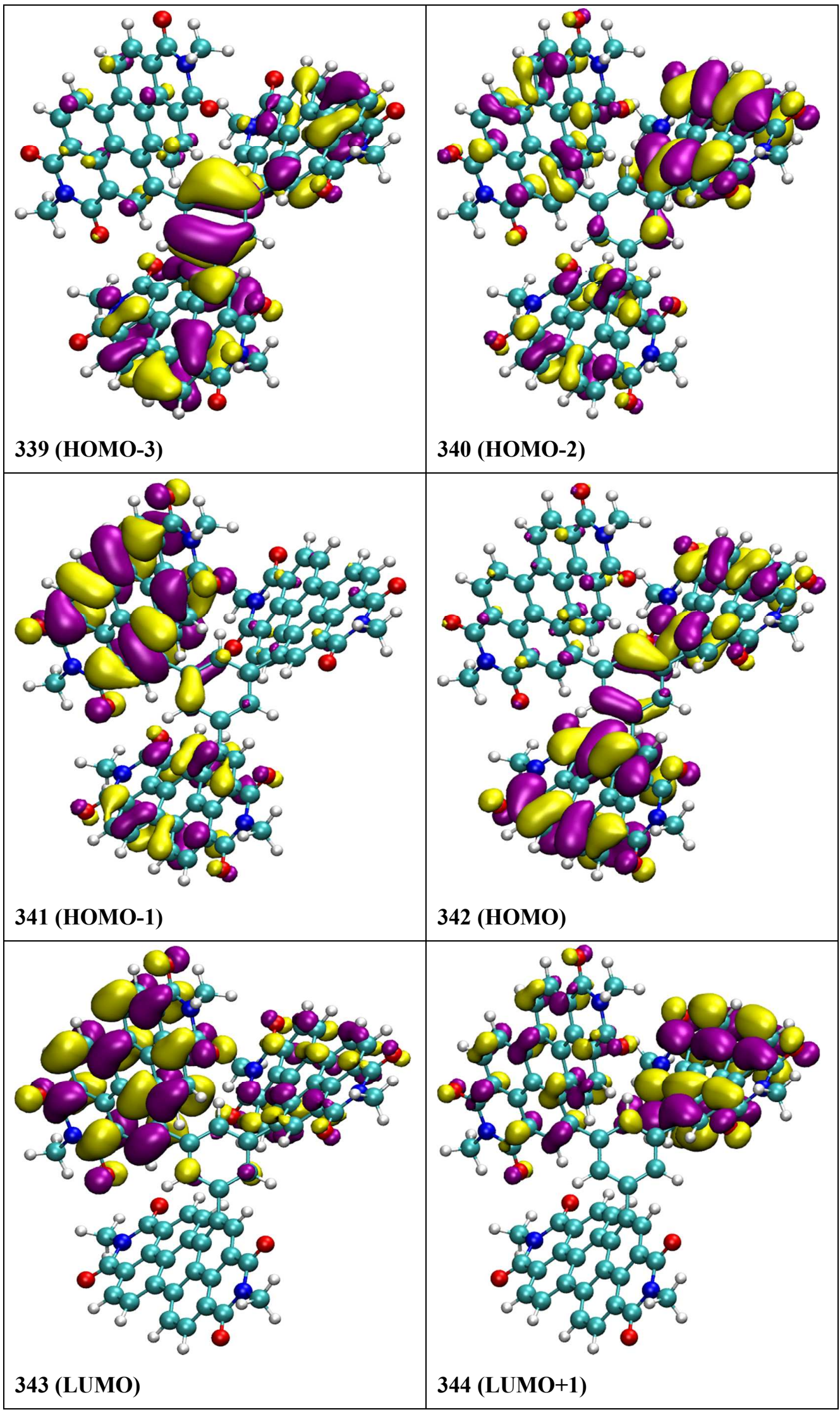




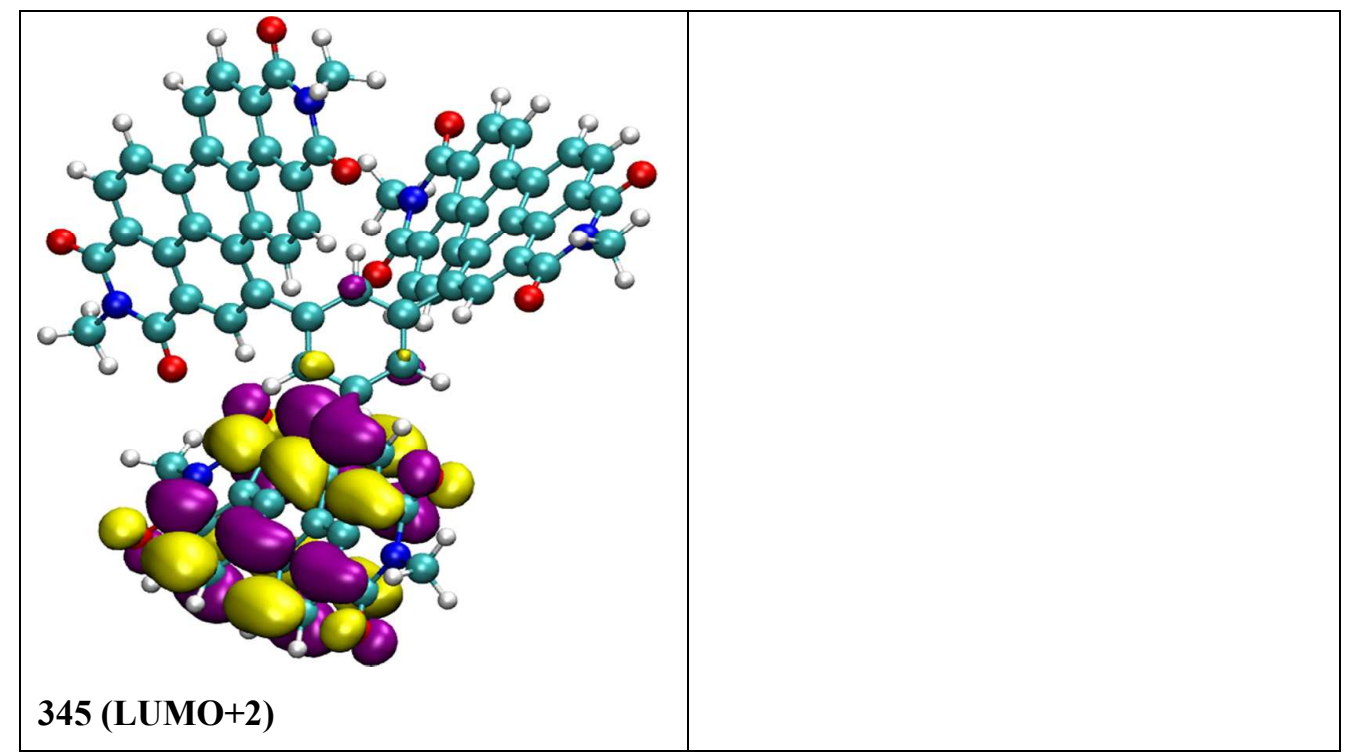

Figure S5. Visualisations of the most important frontier MOs for understanding the electronic behavior of compound $\mathbf{3 a}$.

Transistor Device Fabrication and Characterization: The substrates used here were successively cleaned with pure water, piranha solution $\left(\mathrm{H}_{2} \mathrm{SO}_{4} / \mathrm{H}_{2} \mathrm{O}_{2}=7: 3\right)$, pure water, pure isopropyl alcohol, and finally blown dry with high-purity nitrogen gas. Treatment of the $\mathrm{Si} / \mathrm{SiO}_{2}$ wafers with OTS was carried out by the vapor-deposition method. The clean wafers were dried under vacuum at $90{ }^{\circ} \mathrm{C}$ for $0.5 \mathrm{~h}$ in order to eliminate the influence of moisture. When the temperature decreased to $70{ }^{\circ} \mathrm{C}$, a small drop of OTS was placed around the wafers. Subsequently, this system was heated to $120^{\circ} \mathrm{C}$ and maintained for $2 \mathrm{~h}$ under vacuum.

Micro-/nano-crystals were fabricated by the use of a dilute $\mathrm{CHCl}_{3}$ solution $(0.01$ $\mathrm{mg} \mathrm{mL}{ }^{-1}$ ). Bottom-gate top-contact OFETs based on the Micro-/nano-crystals were constructed on an OTS modified $\mathrm{Si} / \mathrm{SiO}_{2}$ substrate (n-type $\mathrm{Si}$ wafer containing 300 nm-thick $\mathrm{SiO}_{2}$ ) using an "organic ribbon mask" technique. Prior to the self-assembly of polymer nanowires, the OTS modified $\mathrm{Si} / \mathrm{SiO}_{2}$ substrate was cleaned with pure n-hexane, pure chloroform, and pure isopropyl alcohol. Then, polymer nanowires were produced on $\mathrm{Si} / \mathrm{SiO}_{2}$ substrates through drop casting. Subsequently, $40 \mathrm{~nm}$ thick source and drain electrodes were deposited on the nanowires by thermal evaporation with an organic ribbon as the mask. Electrical characteristics of the devices were 
recorded with a Keithley 4200-SCS semiconductor parameter analyzer and a Micromanipulator 6150 probe station in a glove box at room temperature. The mobilities were calculated from the saturation region with the following equation: $I_{\mathrm{DS}}$ $=(W / 2 L) C_{\mathrm{i}} \mu\left(V_{\mathrm{G}}-V_{\mathrm{T}}\right)^{2}$, where $I_{\mathrm{DS}}$ is the drain-source current, $\mathrm{W}$ is the channel width, $\mathrm{L}$ is the channel length, $\mu$ is the field-effect mobility, $C_{\mathrm{i}}$ is the capacitance per unit area of the gate dielectric layer, and $V_{\mathrm{G}}$ and $V_{\mathrm{T}}$ are the gate voltage and threshold voltage, respectively. This equation defines the important characteristics of electron mobility $(\mu)$, on/off ratio $\left(I_{\text {on/off }}\right)$, and threshold voltage $\left(V_{\mathrm{T}}\right)$, which could be deduced by the equation from the plot of current-voltage.

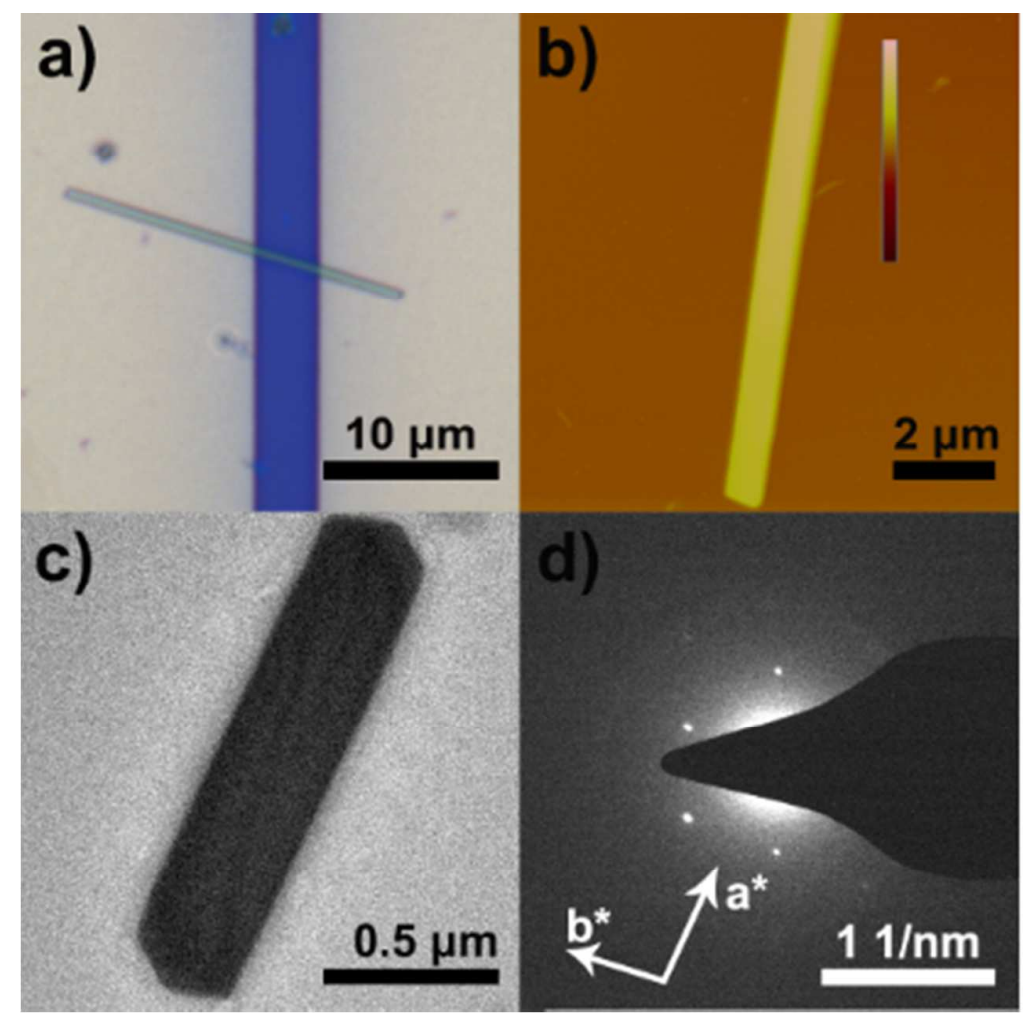

Figure S6. a) Bright field optical microscopy image for $\mathbf{4 b}$. b) The corresponding AFM image of $\mathbf{4 b}$ micro-/nano-crystal. c) TEM image of $\mathbf{4 b}$ micro-/nano-crystal and its corresponding d) SAED patterns. 


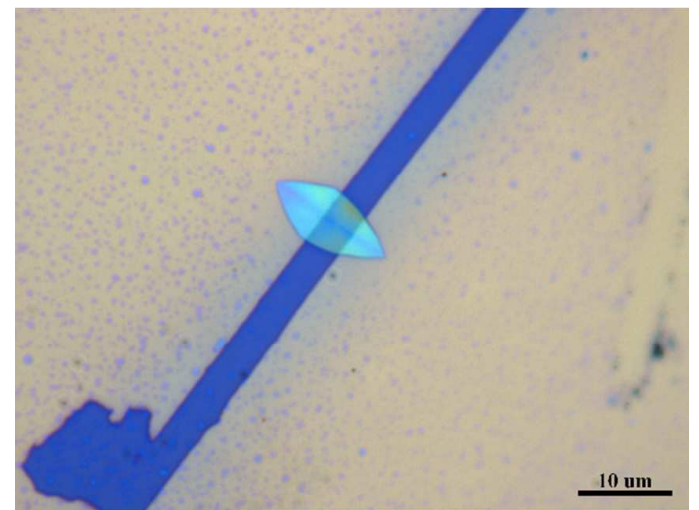

Figure S7. Bright field optical microscopy image for $\mathbf{6 a}$. 
Photovoltaic Device Fabrication and Characterization: The OSC devices were fabricated using a conventional configuration of ITO/ PEDOT:PSS/active layer/Ca/Al. Prepatterned ITO-coated glass was cleaned with detergent, ultrasonicated in deionized water, acetone and isopropanol for $20 \mathrm{~min}$, respectively; subsequently dried in an oven overnight. The substrates were treated by ultraviolet-ozone for $20 \mathrm{~min}$ before using. PEDOT:PSS (Heraeus Clevios P VA A 4083) was spin-cast onto the ITO substrate from aqueous solution at 4000 r.p.m for $40 \mathrm{~s}$ (the thickness is about $40 \mathrm{~nm}$ ), and then dried at $150{ }^{\circ} \mathrm{C}$ for $10 \mathrm{~min}$ in air. The PDBT-T1: 4a and PDBT-T1: 6a blend solutions were prepared in $o$-DCB with different mass ratios and DIO concentrations (PDBT-T1 concentration is fixed at $7 \mathrm{mg} / \mathrm{mL}$ ). Active layers were spin-coated on top of PEDOT:PSS layer at 1000 r.p.m. for 40s followed by thermal annealing at $100{ }^{\circ} \mathrm{C}$ for 5 min to obtain the optimal thickness of $\sim 100 \mathrm{~nm}$ (measured on a Bruker Dektak $\mathrm{XT}$ profilometer) in a $\mathrm{N}_{2}$ glove box. A thin $\mathrm{Ca}$ layer $(10 \mathrm{~nm})$ and $\mathrm{Al}(100 \mathrm{~nm})$ were evaporated through a shadow mask under a vacuum about $5 \times 10^{-6} \mathrm{~Pa}$. The active area is $4.50 \mathrm{~mm}^{2}$. During the measurement, an aperture with the area of $3.14 \mathrm{~mm}^{2}$ was used. Device current density-voltage $(J-V)$ curves were tested using a Keithley 2400 Source Measure Unit in the glove box. The photocurrent was measured under AM 1.5G solar simulator (Class AAA solar simulator, Model 94063A, Oriel) with an irradiation intensity of $100 \mathrm{~mW} / \mathrm{cm}^{2}$. The light intensity was calibrated using a standard Si solar cell and a readout meter (Model 91150V, Newport). EQE spectra were acquired from a QEAX10 Solar Cell EQE measurement system (PV measurement, Inc.). All the fabrication processes were performed inside a controlled atmosphere of $\mathrm{N}_{2}$-filled glove box. 

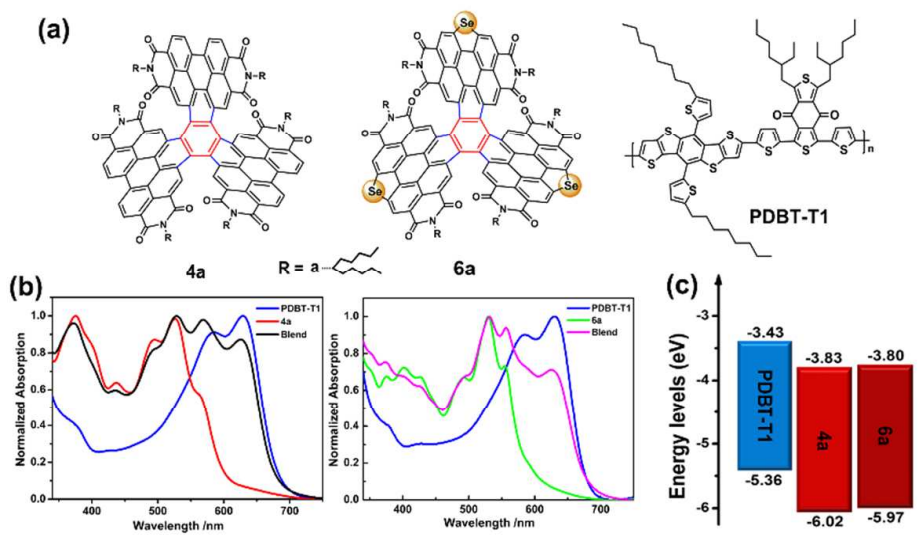

Figure S8. (a) Chemical structures of compounds 4a, 6a and PDBT-T1. (b) Normalized UV-vis absorption spectra of neat PDBT-T1, 4a, 6a, PDBT-T1: 4a blend $(1: 1, w / w)$ and PDBT-T1: 6a blend $(1: 1, w / w)$ films. (c) Energy levels of diagrams of PDBT-T1, 4a and 6a.

Table S2. Summary of Device Parameters of Solar Cells Based on PDBT-T1: 4a and PDBT-T1: 6a with Different D/A Weight Ratios.

\begin{tabular}{ccccccc}
\hline Active layer & $\begin{array}{c}\mathrm{D} / \mathrm{A} \\
\text { Ratio }\end{array}$ & $\begin{array}{c}\mathrm{V}_{\mathrm{OC}} \\
{[\mathrm{V}]}\end{array}$ & $\begin{array}{c}J_{\mathrm{SC}} \\
{\left[\mathrm{mA} / \mathrm{cm}^{2}\right]}\end{array}$ & $\begin{array}{c}\mathrm{FF} \\
{[\%]}\end{array}$ & $\begin{array}{c}\mathrm{PCE}^{a} \\
{[\%]}\end{array}$ & $\begin{array}{c}\mathrm{PCE}_{\max } \\
{[\%]}\end{array}$ \\
\hline & $1.5: 1$ & $0.965 \pm 0.004$ & $11.64 \pm 0.12$ & $63.2 \pm 0.6$ & $7.10 \pm 0.08$ & 7.18 \\
PDBT-T1:4a & $1: 1$ & $0.961 \pm 0.003$ & $11.71 \pm 0.05$ & $64.8 \pm 0.6$ & $7.28 \pm 0.07$ & 7.34 \\
& $1: 1.5$ & $0.963 \pm 0.003$ & $11.04 \pm 0.19$ & $63.1 \pm 0.8$ & $6.71 \pm 0.08$ & 6.78 \\
\hline PDBT-T1:6a & $1.2: 1$ & $1.002 \pm 0.003$ & $11.22 \pm 0.24$ & $65.8 \pm 0.7$ & $7.39 \pm 0.20$ & 7.72 \\
& $1: 1.2$ & $1.007 \pm 0.003$ & $12.32 \pm 0.17$ & $63.9 \pm 0.8$ & $7.93 \pm 0.09$ & 8.05 \\
& & & $12.24 \pm 0.18$ & $62.4 \pm 1.0$ & $7.64 \pm 0.12$ & 7.79 \\
\hline
\end{tabular}

${ }^{a}$ The average PCE value was calculated from six devices for each condition 

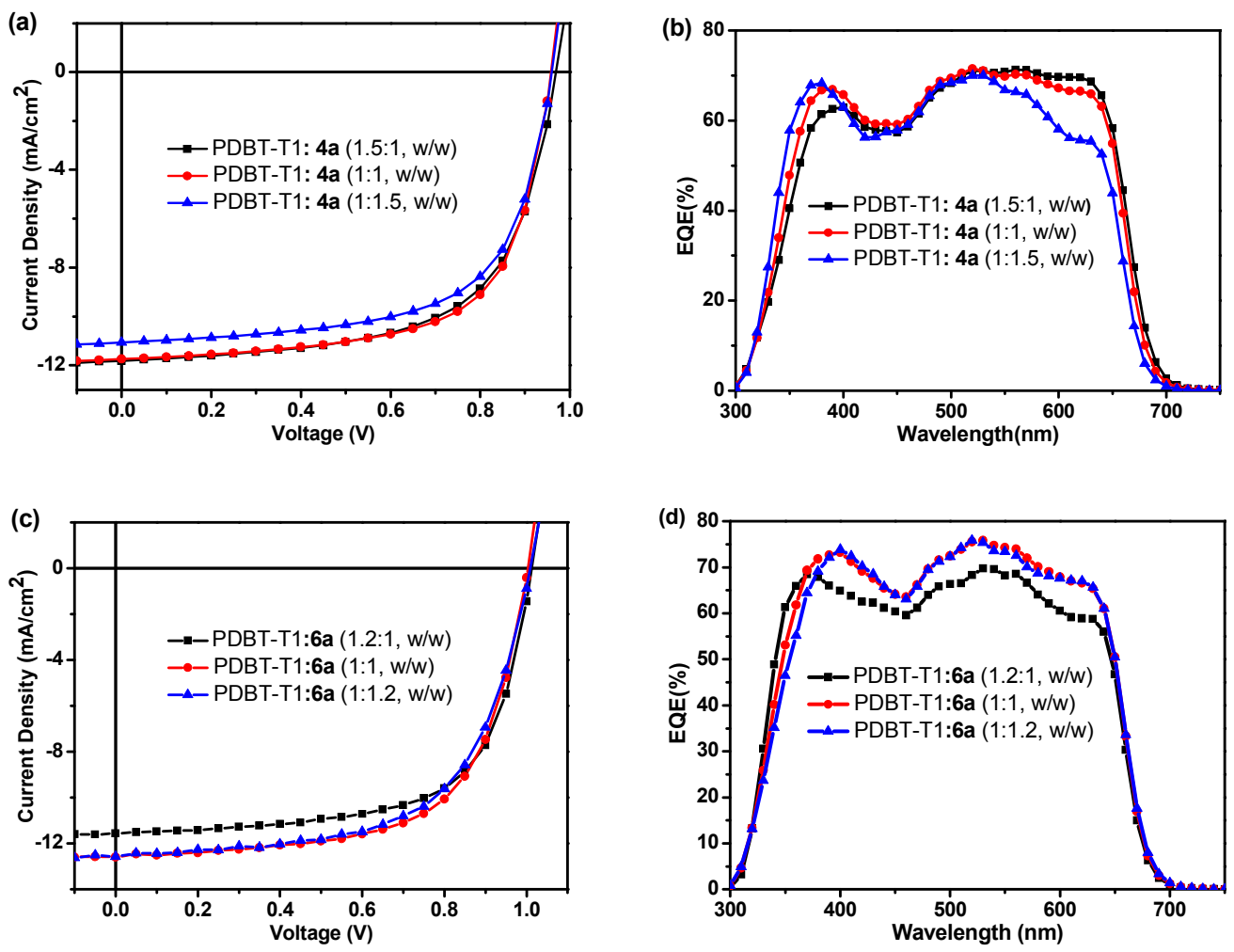

Figure S9. (a, c) $J$-V curves of solar cells based on PDBT-T1:4a and PDBT-T1:6a with different weight ratios and $(b, d)$ the corresponding EQE spectra.

Table S3. Summary of Device Parameters of PDBT-T1: 4a Solar Cells with Different DIO Concentrations.

\begin{tabular}{cccccc}
\hline $\begin{array}{c}\mathrm{DIO} \\
{[\%]}\end{array}$ & $\begin{array}{c}\mathrm{V}_{\mathrm{OC}} \\
{[\mathrm{V}]}\end{array}$ & $\begin{array}{c}J_{\mathrm{SC}} \\
{\left[\mathrm{mA} / \mathrm{cm}^{2}\right]}\end{array}$ & $\begin{array}{c}\mathrm{FF} \\
{[\%]}\end{array}$ & $\begin{array}{c}\mathrm{PCE}^{a} \\
{[\%]}\end{array}$ & $\begin{array}{c}\mathrm{PCE}_{\max } \\
[\%)]\end{array}$ \\
\hline 0 & $0.961 \pm 0.003$ & $11.71 \pm 0.05$ & $64.8 \pm 0.6$ & $7.28 \pm 0.07$ & 7.34 \\
0.25 & $0.968 \pm 0.002$ & $12.01 \pm 0.19$ & $70.1 \pm 0.3$ & $8.15 \pm 0.14$ & 8.28 \\
0.5 & $0.967 \pm 0.005$ & $11.65 \pm 0.16$ & $70.2 \pm 1.2$ & $7.90 \pm 0.21$ & 8.12 \\
1 & $0.956 \pm 0.003$ & $11.18 \pm 0.11$ & $66.0 \pm 0.5$ & $7.06 \pm 0.13$ & 7.18 \\
\hline
\end{tabular}

${ }^{a}$ The average PCE value was calculated from six devices for each condition 
Table S4. Summary of Device Parameters of PDBT-T1: 6a Solar Cells with Different DIO Concentrations.

\begin{tabular}{cccccc}
\hline $\begin{array}{c}\mathrm{DIO} \\
{[\%]}\end{array}$ & $\begin{array}{c}\mathrm{V}_{\mathrm{OC}} \\
{[\mathrm{V}]}\end{array}$ & $\begin{array}{c}J_{\mathrm{SC}} \\
{\left[\mathrm{mA} / \mathrm{cm}^{2}\right]}\end{array}$ & $\begin{array}{c}\mathrm{FF} \\
{[\%]}\end{array}$ & $\begin{array}{c}\mathrm{PCE}^{a} \\
{[\%]}\end{array}$ & $\begin{array}{c}\mathrm{PCE}_{\max } \\
[\%)]\end{array}$ \\
\hline 0 & $1.007 \pm 0.003$ & $12.32 \pm 0.17$ & $63.9 \pm 0.8$ & $7.93 \pm 0.09$ & 8.05 \\
0.5 & $0.998 \pm 0.004$ & $12.36 \pm 0.19$ & $71.2 \pm 0.4$ & $8.78 \pm 0.12$ & 8.90 \\
0.75 & $1.001 \pm 0.004$ & $12.53 \pm 0.19$ & $71.7 \pm 0.4$ & $8.98 \pm 0.12$ & 9.28 \\
1 & $0.998 \pm 0.003$ & $11.64 \pm 0.14$ & $70.5 \pm 0.8$ & $8.18 \pm 0.07$ & 8.27
\end{tabular}

${ }^{a}$ The average PCE value was calculated from six devices for each condition
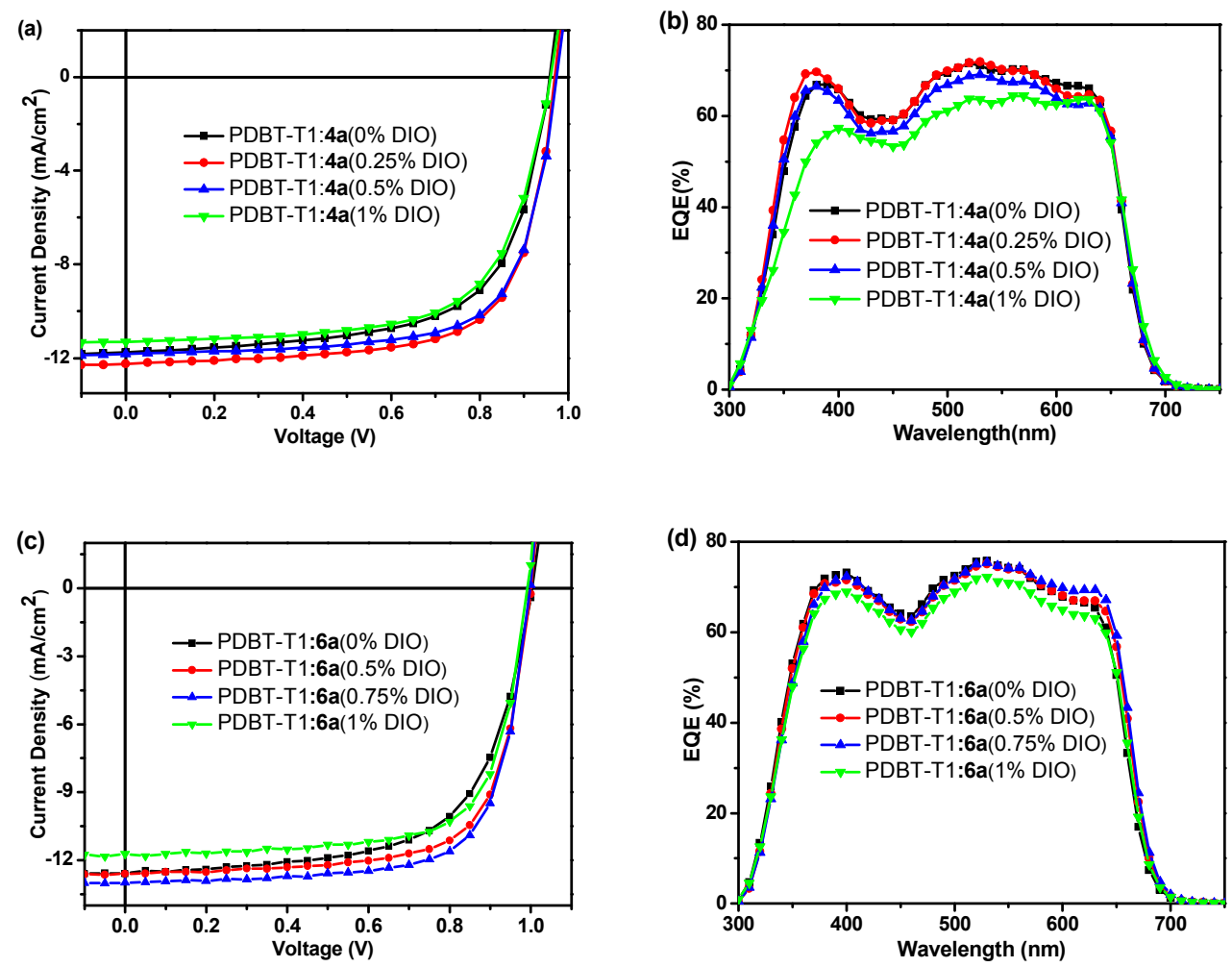

Figure S10. (a, c) $J$-V curves of solar cells based on PDBT-T1:4a and PDBT-T1:6a with different DIO concentrations and $(b, d)$ the corresponding EQE spectra.

Carrier Mobility Measurement: Carrier mobility was measured by space charge limit current (SCLC) method. Electron-only devices were fabricated with a device architecture of ITO/Al/PDBT-T1: acceptor/Al. Hole-only devices were fabricated with a device architecture of ITO/MoOx/PDBT-T1: acceptor/ MoOx. The electron mobilities in neat $4 \mathbf{a}$ and $6 \mathbf{a}$ films were measured with the device architecture of 
ITO/Al/ $4 \mathbf{a}$ or $\mathbf{6 a} / \mathrm{Al}$. The mobility was determined by fitting the dark current to the model of a single carrier SCLC, described by the equation:

$$
J=9 \varepsilon_{0} \varepsilon_{\mathrm{r}} \mu V^{2} / 8 \mathrm{~d}^{3}
$$

where $J$ is the current density, $\varepsilon_{0}$ is the permittivity of free space, $\varepsilon_{\mathrm{r}}$ is the relative dielectric constant of the transport medium, $\mu$ is the charge mobility and $d$ is the active layer thickness. $V=V$ app $-V_{\mathrm{bi}}$, where $V \mathrm{app}$ is the applied voltage, $V_{\mathrm{bi}}$ is the offset voltage ( $V_{\mathrm{bi}}$ is $0 \mathrm{~V}$ here). The carrier mobility can be calculated from the slope of the $J^{0.5} \sim V$ curves.
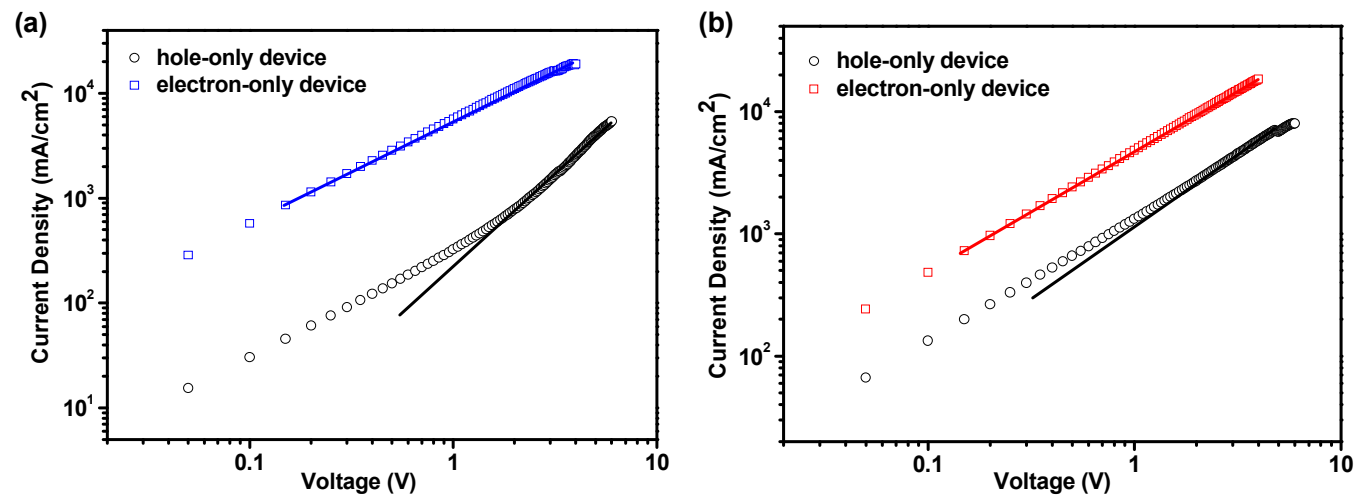

Figure S11. The experimental current density-applied voltage $(J-V)$ characteristics for hole-only device, and electron-only device for (a) PDBT-T1: 4a blend films and (b) PDBT-T1: 6 a blend films.

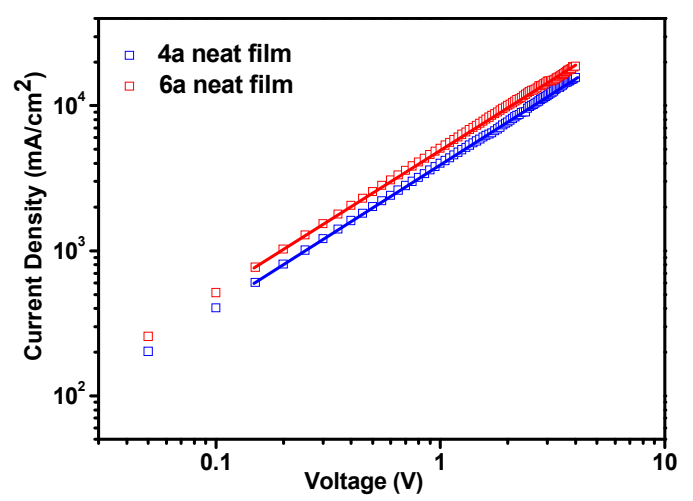

Figure S12. The experimental current density-applied voltage $(J-V)$ characteristics for the $4 \mathbf{a}$ and $\mathbf{6 a}$ neat films. 

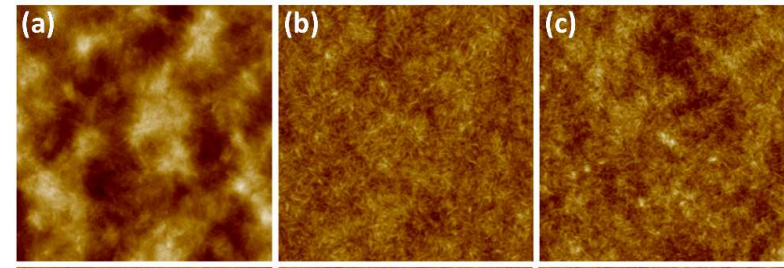

(d)

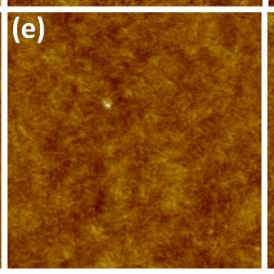

(f)
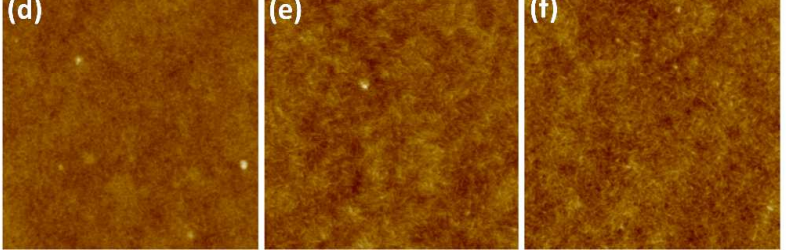

$20.0 \mathrm{~nm}$

Figure S13. Tapping-mode AFM height images $(2 \times 2 \mu \mathrm{m})$ of (a) 4a neat film, PDBT-T1:4a blend films (b) without and (c) with 0.25\% DIO, (d) 6a neat film, and PDBT-T1:6a blend films (e) without and (f) with $0.75 \%$ DIO.

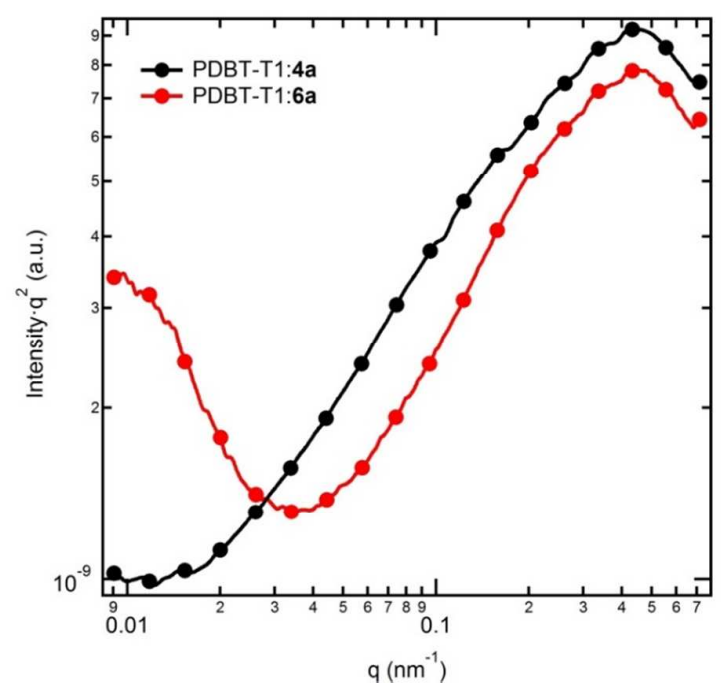

Figure S14. R-SoXS profiles for PDBT-T1:4a blend film with $0.25 \%$ DIO and for PDBT-T1:6a blend film with $0.75 \%$ DIO. 


\section{$\underline{\text { References }}$}

(1) (a) Sun, D.; Meng, D.; Cai, Y.; Fan, B.; Li, Y.; Jiang, W.; Huo, L.; Sun, Y.; Wang, Z. J. Am. Chem. Soc. 2015, 137, 11156-11162. (b) Meng, D.; Sun, D.; Zhong, C.; Liu, T.; Fan, B.; Huo, L.; Li, Y.; Jiang, W.; Choi, H.; Kim, T.; Kim, J. Y.; Sun, Y.; Wang, Z.; Heeger, A. J. J. Am. Chem. Soc. 2016, 138, 375-380.

(2) M. J. Frisch, G. W. Trucks, H. B. Schlegel, G. E. Scuseria, M. A. Robb, J. R. Cheeseman, G. Scalmani, V. Barone, B. Mennucci, G. A. Petersson, H. Nakatsuji, M. Caricato, X. Li, H. P. Hratchian, A. F. Izmaylov, J. Bloino, G. Zheng, J. L. Sonnenberg, M. Hada, M. Ehara, K. Toyota, R. Fukuda, J. Hasegawa, M. Ishida, T. Nakajima, Y. Honda, O. Kitao, H. Nakai, T. Vreven, Jr. Montgomery, J. A., J. E. Peralta, F. Ogliaro, M. Bearpark, J. J. Heyd, E. Brothers, K. N. Kudin, V.

N. Staroverov, R. Kobayashi, J. Normand, K. Raghavachari, A. Rendell, J. C. Burant, S. S. Iyengar, J. Tomasi, M. Cossi, N. Rega, M. J. Millam, M. Klene, J. E. Knox, J. B. Cross, V. Bakken, C. Adamo, J. Jaramillo, R. Gomperts, R. E. Stratmann, O. Yazyev, A. J. Austin, R. Cammi, C. Pomelli, J. W. Ochterski, R. L. Martin, K. Morokuma, V. G. Zakrzewski, G. A. Voth, P. Salvador, J. J. Dannenberg, S. Dapprich, A. D. Daniels, O. Farkas, J. B. Foresman, J. V. Ortiz, J. Cioslowski, and D. Fox, 2009. Gaussian 09, Revision D.01, Gaussian, Inc., Wallingford, CT.

(3) C. Adamo and V. Barone. J. Chem. Phys., 1999, 110, 6158-6170.

(4) X. Li and M. J. Frisch, J. Chem. Theory and Comput., 2006, 2, 835-839.

(5) J. Simons, P. Jørgensen, H. Taylor, and J. Ozment, J. Phys. Chem., 1983, 87, 2745-2753.

(6) J. Tomasi, B. Mennucci, and R. Cammi, Chem. Rev., 2005, 105, 2999-3093. 